\title{
A Survey of \\ Nuclear-Explosive \\ Prompt Diagnostics
}

Paul J. Ebert

March 25, 1986 


\section{DISCLAIMER}

This report was prepared as an account of work sponsored by an agency of the United States Government. Neither the United States Government nor any agency Thereof, nor any of their employees, makes any warranty, express or implied, or assumes any legal liability or responsibility for the accuracy, completeness, or usefulness of any information, apparatus, product, or process disclosed, or represents that its use would not infringe privately owned rights. Reference herein to any specific commercial product, process, or service by trade name, trademark, manufacturer, or otherwise does not necessarily constitute or imply its endorsement, recommendation, or favoring by the United States Government or any agency thereof. The views and opinions of authors expressed herein do not necessarily state or reflect those of the United States Government or any agency thereof. 


\section{DISCLAIMER}

Portions of this document may be illegible in electronic image products. Images are produced from the best available original document. 
UCRL-53724

Distribution Category UC-37

UCRL --53724

DE87 002738

\title{
A Survey of Nuclear-Explosive Prompt Diagnostics
}

\author{
Paul J. Ebert
}

Manuscript date: March 25, 1986

DISCLAIMER

\begin{abstract}
This report was prepared as an account of work sponsored by an agency of the United States Government. Neither the United States Government nor any agency thereof, nor any of their employees, makes any warranty, express or implied, or assumes any legal liability or responsibility for the accuracy, completeness, or usefulness of any information, apparatus, product, or process disclosed, or represents that its use would not infringe privately owned rights. Reference herein to any specific commercial product, process, or service by trade name, trademark, manufacturer, or otherwise does not necessarily constitute or imply its endorsement, recommendation, or favoring by the United States Government or any agency thereof. The views and opinions of authors expressed herein do not necessarily state or reflect those of the United States Government or any agency thereof.
\end{abstract}

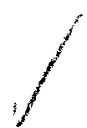

\section{LAWRENCE LIVERMORE NATIONAL LABORATORY University of California - Livermore, California 94550}

Available from: National Technical Information Service - U.S. Department of Comm
5285 Port Royal Road $\bullet$ Springfield, VA $22161 \bullet$ A02 $\quad$ (Microfiche A01) 


\section{Contents}

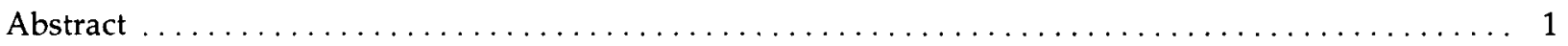

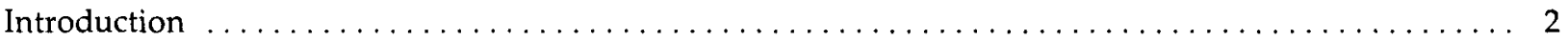

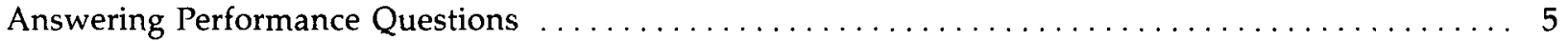

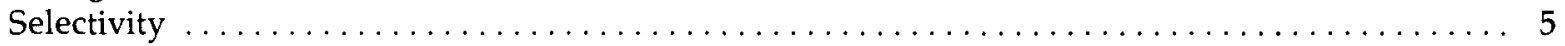

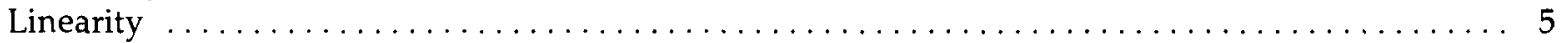

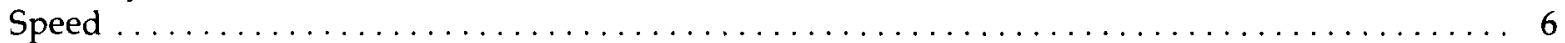

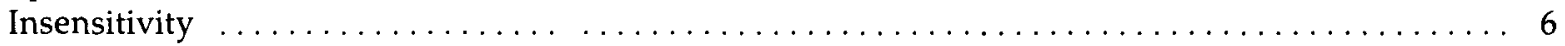

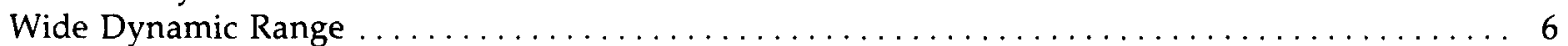

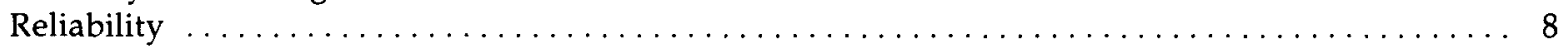

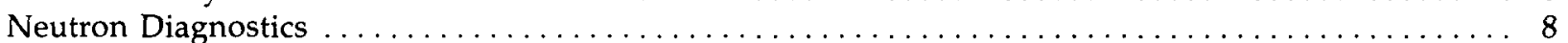

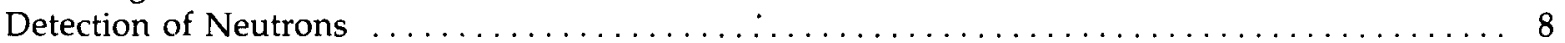

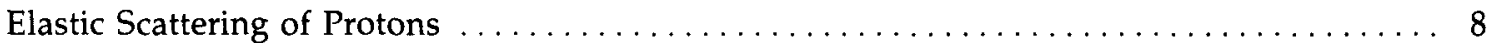

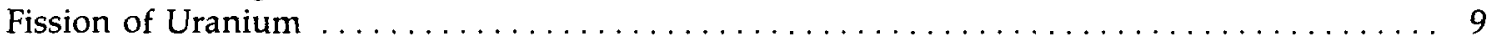

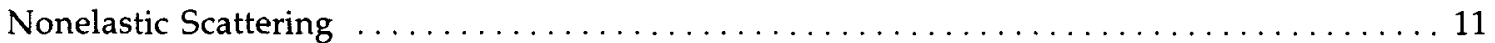

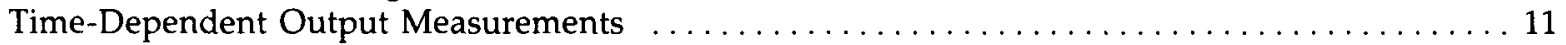

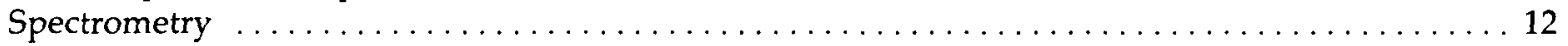

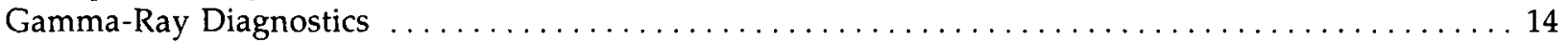

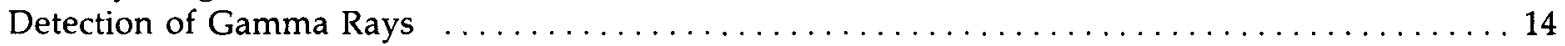

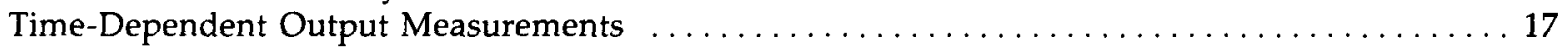

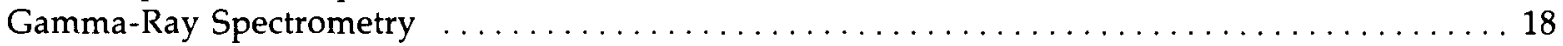

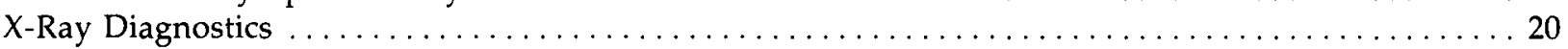

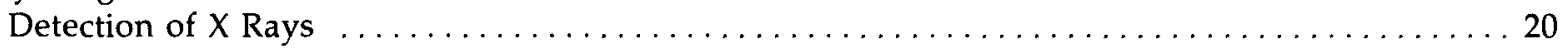

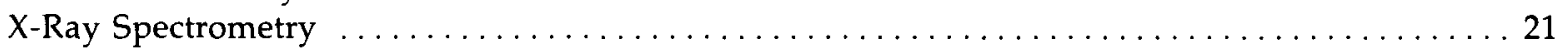

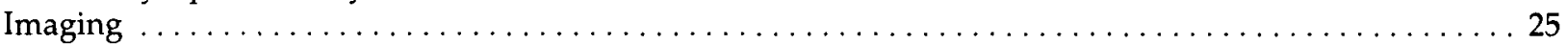

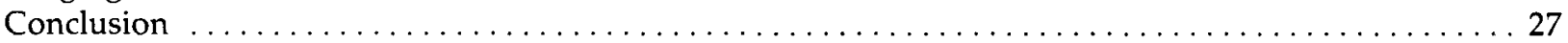




\title{
A Survey of \\ Nuclear-Explosive \\ Prompt Diagnostics
}

\begin{abstract}
The author surveys nuclear-explosive prompt diagnostics techniques and equipment. These techniques and equipment have been developed to answer nuclearexplosive performance questions. The techniques and equipment must be selective in radiation sensitivity, linear in calibration, fast, insensitive to strong signals, wide in dynamic range, and reliable. Diagnostic techniques and equipment measure neutron, gamma-ray, and x-ray emissions, as well as aid in the determination of the physical location of the production of radiation through imaging.

The high cost of nuclear experiments will continue to encourage the development of sophisticated techniques to gain as much information as possible from each experiment.
\end{abstract}




\section{Introduction}

Nuclear explosives research is required to meet a number of national security objectives. This research begins with concepts that are carefully modeled in many computer calculations. Experiments are formulated and conducted to test the soundness of the concepts. Data are analyzed and experimental results interpreted so that the models can be perfected and improved concepts might unfold. Most nuclear "tests" are conducted with computers because this is faster and cheaper. However, as any scientist knows, contact with reality must be maintained; thus, full-scale tests are conducted in Nevada. Since these involve a number of hazards and experimental conditions not encountered in other areas of research, unusual expertise is required. As a result of ongoing test activity, a unique scientific discipline has emerged-nuclear-explosive prompt diagnostics. This report surveys this discipline by reviewing how scientific data on the performance of a nuclear explosive are obtained. Emphasis is placed on detectors and experimental techniques since these are central in obtaining high-quality scientific information in a cost-effective manner.

Most scientists and engineers are unfamiliar with the "big-physics" field of nuclear testing. The bigness of nuclear testing is illustrated in Fig. 1, which shows a nuclear explosive experiment being prepared for lowering downhole. This package, holding the nuclear explosive, diagnostic instruments, and auxiliary shielding material, just fits into a $2.4-\mathrm{m}(8-\mathrm{ft})$ diameter hole. Typically, the hole is over $300 \mathrm{~m}(1000 \mathrm{ft})$ deep. The package may be over $30 \mathrm{~m}$ (100 ft) long and weigh several hundred thousand kilograms. Portable recording and control stations are positioned hundreds of meters from the emplacement hole. When the requisite depth is reached, the emplacement hole is backfilled for detonation. The overall test configuration is illustrated in Fig. 2.

An unclassified description of the explosion process is presented here to help the reader appreciate the complexities involved in acquiring nuclear-explosive performance data. Figure 3 schematically illustrates a fission device. A subcritical mass of fissile material is surrounded by chemical explosive. Upon detonation of the chemical explosive, the fissile material assembles from subcritical to super-prompt-critical, thereby initiating the fission chain reaction, an exponential growth process. The neutron population rapidly multiplies, and nuclear energy is produced. Thermonuclear reactions associated with the chain re- action can increase the neutron multiplication rate to a higher level, thereby adding significantly to the energy production. This is called boosting. The nuclear energy produced is far in excess of the chemical energy expended to assemble the nuclear component. Within a few microseconds after initiation, everything in the vicinity of the device, including the diagnostic instruments, is vaporized as the nuclear fireball expands into the surrounding medium.

In an underground nuclear explosion, most of the energy released is blast energy. However, a few percent of the energy is released in prompt, penetrating radiation (neutrons and gamma rays) that is measured to diagnose device performance. To appreciate the difficulty of nuclear-explosive diagnostics, let us consider a fission explosive yielding 1 kiloton $(\mathrm{kt})$. By definition $1 \mathrm{kt}$ $=10^{12} \mathrm{cal}=4.2 \times 10^{12} \mathrm{~J}$. Each fission produces $200 \mathrm{MeV}$ or $3.2 \times 10^{-11} \mathrm{~J}$. Therefore $\mathrm{N}_{\mathrm{F}}$, the number of fissions that take place in a $1-\mathrm{kt}$ chain reaction, is $1.3 \times 10^{23}$. This is approximately 53 e-foldings or generations. The entire chain reaction is over in about $5 \times 10^{-7} \mathrm{~s}$. Thus $\tau$, the generation time, is about $10^{-8} \mathrm{~s}$. $\tau$ is the time required for the number of fissions (and hence the neutron population) to increase by a factor of $e$ and is related to the velocity of fast neutrons produced in fission, among other things. For the exponential chain reaction, the peak rate is given by $\dot{\mathrm{N}}_{\text {peak }}=$ $\mathrm{N}_{\mathrm{F}} / \tau=10^{31}$ fissions/s. This is approximately the peak production rate of both neutrons and gamma rays within the explosive.

Not only are nuclear explosions incredibly fast, they are also intensely hot. That the explosive temperature is high can be seen as follows. A canonical nuclear device contains $10 \mathrm{~kg}$ of plutonium $(\mathrm{Pu})$. There are approximately $2.5 \times 10^{25}$ atoms of $\mathrm{Pu}$ in $10 \mathrm{~kg}$ that share $4.2 \times 10^{12} \mathrm{~J}$. The energy shared per atom is $1.7 \times 10^{-13} \mathrm{~J} \simeq 1 \mathrm{MeV}$. Clearly, the atoms must be highly ionized and, therefore, at high temperature. A lot of the energy released is expended in ionization, as well as in heating electrons, which have very little mass but high heat capacity. We can estimate the temperature from the expression $\mathrm{T}=\mathrm{Y} / \mathrm{mC}_{\mathrm{v}}$, where $\mathrm{Y}$ is the nuclear yield, $m$ is the mass, and $C_{v}$ is the heat capacity. Assuming $C_{v}=10 \mathrm{cal} / \mathrm{g} \cdot \mathrm{K}$, we obtain $\mathrm{T}=10^{7} \mathrm{~K}$. From the Stefan-Boltzmann Law, $\mathrm{W}=$ $\sigma \mathrm{T}^{4}$, where $\sigma=5.7 \times 10^{-12} \mathrm{~J} / \mathrm{s} \cdot \mathrm{cm}^{2} \cdot \mathrm{K}^{4}$, we can calculate the amount of thermal radiation emitted per square centimeter of source, $W=5.7 \times 10^{16}$ $\mathrm{J} / \mathrm{s} \cdot \mathrm{cm}^{2}$. This radiation is emitted in a Planckian 


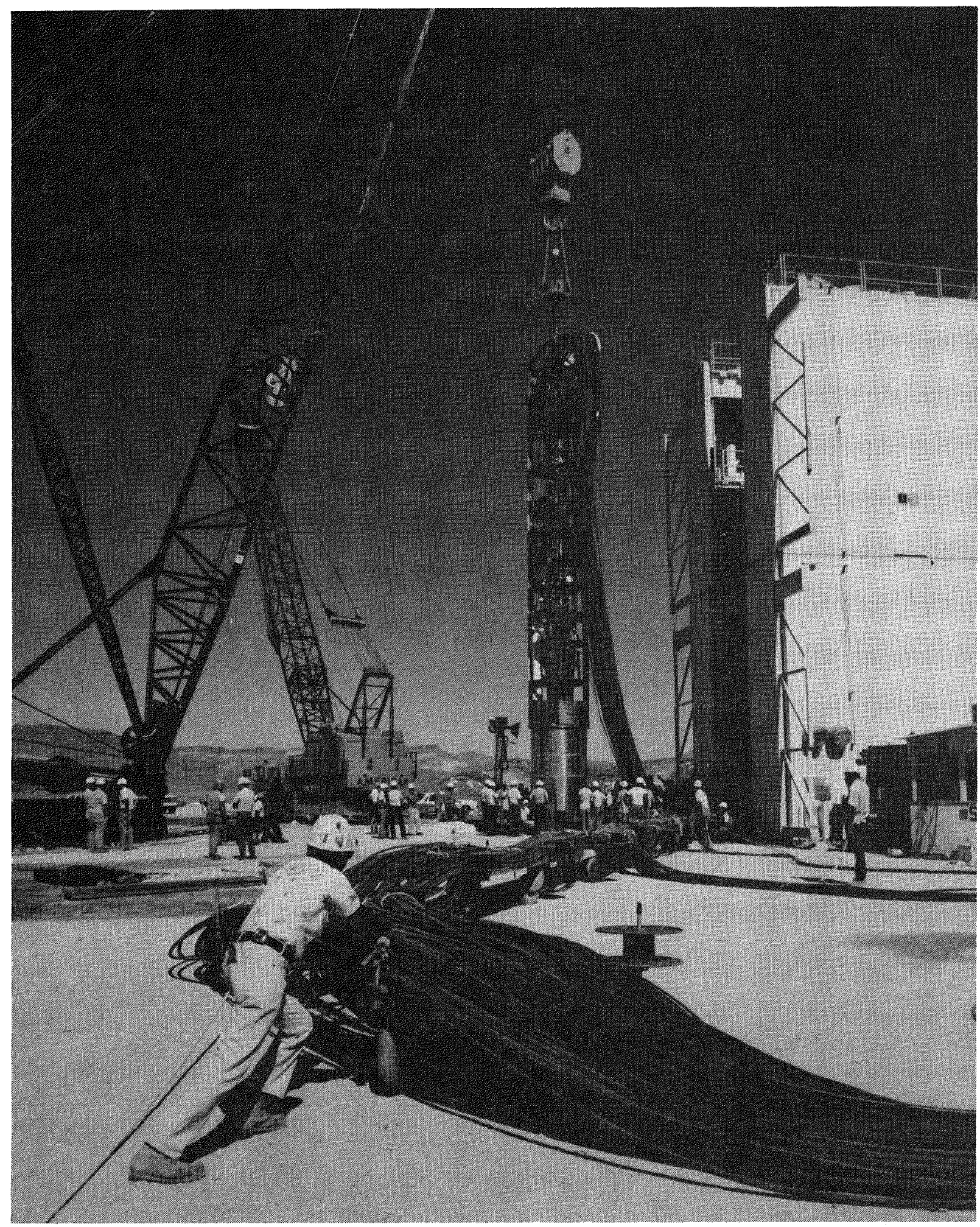

Figure 1. The nuclear experiment being prepared for lowering downhole is held by a crane with 1000-ton capacity. The nuclear explosive is housed in the shrouded region. Diagnostics experiments are positioned in the heavy canister structure and view the device through beam lines that keep air or dirt and moisture out of the line of sight. As the package is initially lowered, the canister is covered with steel, and auxiliary shielding materials are added to fill the voids between beam lines. 


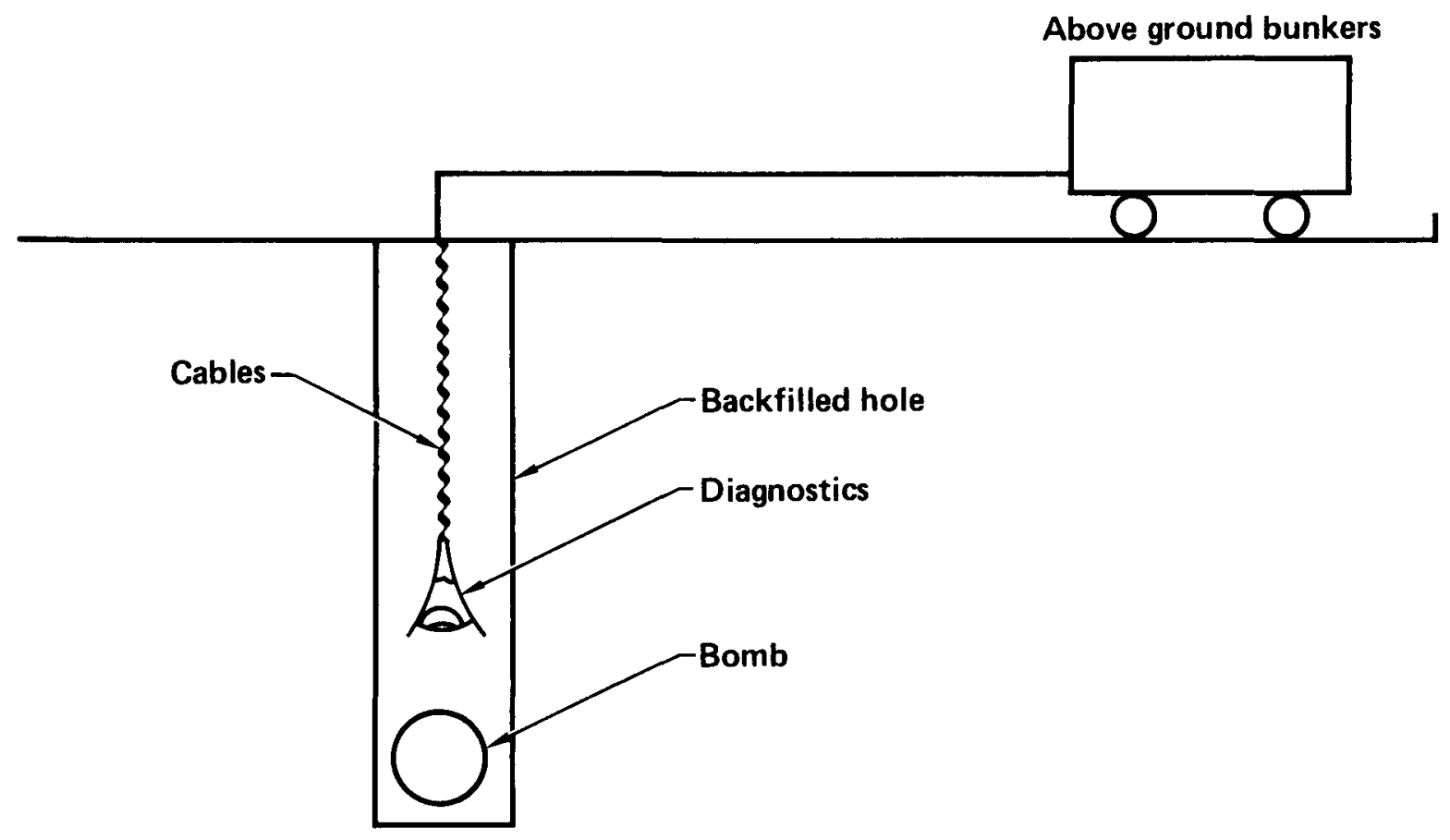

Figure 2. Schematic diagram of the underground test configuration. The typical emplacement hole is $2.4 \mathrm{~m}$ in diameter and is over $300 \mathrm{~m}$ deep. The surrounding earth, backfill material, and local shielding in the diagnostic canister shield the cables and significantly reduce background. In atmospheric testing, which was banned in 1962, the cables were exposed to scattered radiation and the bunkers were buried for protection from the blast.

Subcritical assembly

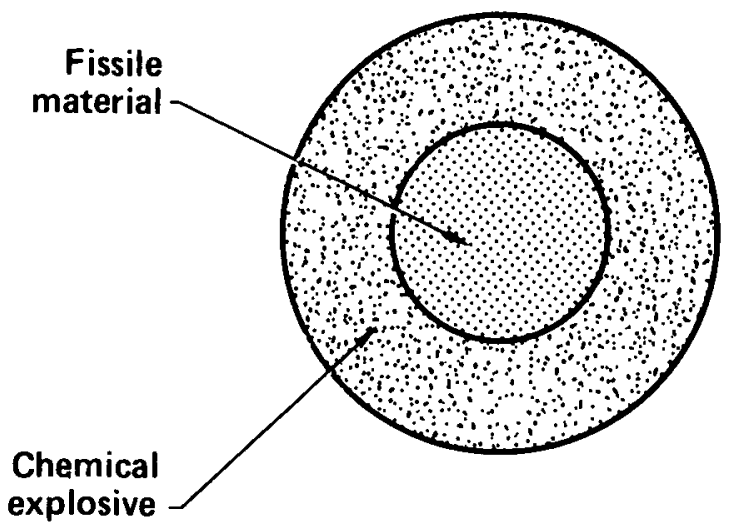

Super-prompt-critical assembly

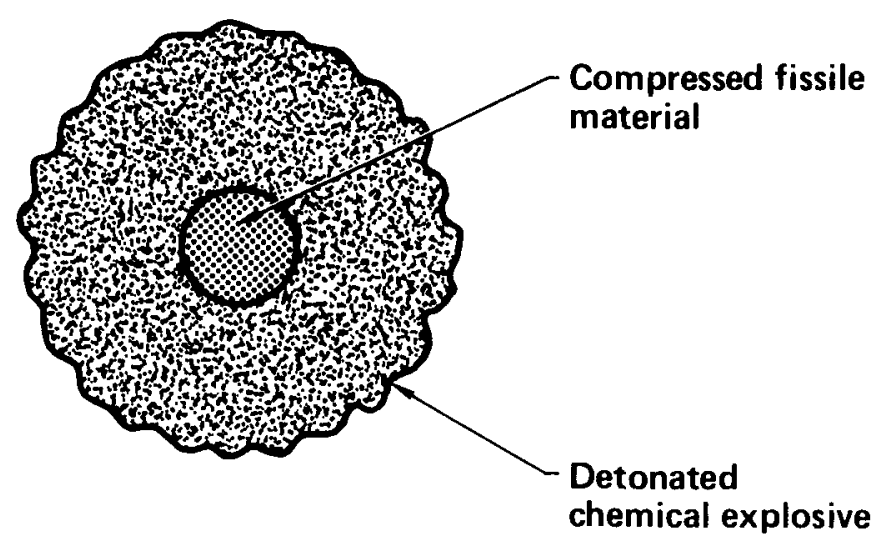

Figure 3. Schematic diagram of a fission explosive. A subcritical mass is assembled to superprompt-critical by the high explosive. This diagram is adapted from Samuel Glasstone and Philip J. Dolan, Effects of Nuclear Weapons, 3rd ed. (U.S. Government Printing Office, Washington, D.C., 1977). 
spectrum for which the most probable energy of the photons is $\mathrm{h} v_{\mathrm{p}}=2.8 \mathrm{kT}$, where $\mathrm{k}$ is Boltzmann's constant $\left(\mathrm{k}=0.86 \times 10^{-7} \mathrm{eV} / \mathrm{K}\right)$ and $\mathrm{T}$ is the absolute temperature. Thus $\mathrm{h} v_{\mathrm{p}}=2.4 \mathrm{keV}$, which is the soft $x$-ray region of the electromagnetic spectrum. The rate at which $x$ rays emanate from $1 \mathrm{~cm}^{2}$ of the $10^{7} \mathrm{~K}$ thermal source is $\left(5.7 \times 10^{16}\right.$ $\left.\mathrm{W} / \mathrm{cm}^{2}\right) \times\left(6 \times 10^{15} \mathrm{keV} / \mathrm{s} \cdot \mathrm{W}\right) \times(1 \times$ ray $/ 2.8 \mathrm{keV})$ $\simeq 10^{32} \mathrm{x}$ rays $/ \mathrm{cm}^{2} \cdot \mathrm{s}$ !
Thus, from very simplistic assumptions, it is easy to see that the source strengths are enormous in comparison with ordinary terrestrial sources. It would be difficult for even the highest-speed laboratory counting system to measure a source $10^{20}$ times smaller. Clearly, conventional laboratory counting techniques must give way to detectors and techniques that generate and measure current pulses of short duration.

\section{Answering Performance Questions}

As stated earlier, extensive calculations are carried out to quantitatively predict device performance. To verify these calculations, a lengthy set of questions about specific phenomena must be answered experimentally. These questions can be functional or scientific in nature and are generally summarized as follows:

- What were the emission rates of neutrons, gamma rays, and $x$ rays?

- How many were produced?

- What were their energy spectra?

- Where were the radiations produced?

By answering these questions, device-component performance as well as overall device performance can be inferred. Common to all radiation measurements, the nature of an unknown source is inferred from signals recorded in a known diagnostic system.

Many textbooks, monographs, and journal articles have been published on radiation measurements and the interaction of radiation with matter. In nuclear-explosive diagnostics, the physical principles of the detectors and methods are generally the same as in other areas of science. Therefore, for more details about the principles of radiation detection, the reader should consult this vast literature. Indeed, many of the instruments and methods described here have already proved useful in laser- and ion-beam fusion, in magnetic fusion, and in diagnostics of high-current particle beams. The explosive source, however, is significantly different in output, strength, and time scale. To operate properly in the underground explosion environment, the diagnostics system must record signals before the detectors are destroyed. They have the properties listed below.

\section{Selectivity}

The detector must select the desired radiation in the presence of large fluxes* of undesired radiation, such as neutrons in the presence of gamma rays. In measuring spectra, there can also be the additional problem of differentiating between energies. Selectivity depends upon the method of detection. As examples, it is possible to differentiate between gamma rays and neutrons using time of flight, and it is possible to detect a relatively small flux of high-energy photons in the presence of a much larger flux of lower-energy photons by the choice of materials, their thicknesses, and magnetic fields. One of the best methods of reducing extraneous backgrounds is to provide enough collimation and shielding so that only the desired part of the explosive can be viewed and only the sensitive volume of the detector is in the direct radiation beam. The earth and auxiliary shielding material surrounding beam pipes also help to reduce scattering into the detector.

\section{Linearity}

The laboratory calibration at low flux levels must be valid for high-flux experiments. A laboratory calibration source may produce a flux which is too high for counting but is barely measurable with a diagnostic detector and a sensitive electrometer. At the other extreme, the flux at $10 \mathrm{~m}$

\footnotetext{
* Flux is defined as particles per unit area per unit time, for example, neutrons $/ \mathrm{cm}^{2} \mathrm{~s}$ We assume isotropic sources, from which the flux, $\phi$, at any distance, $R$, is given by $\phi=\dot{N} / 4 \pi R^{2}$, where $N$ is the source emission rate
} 
from an isotropic source emitting at a peak rate of $10^{31}$ particles/s is approximately $10^{24}$ particles/ $\mathrm{cm}^{2} \cdot \mathrm{s}$. This is sufficient to generate detector current of several thousand amperes, which is well above the linear output capability. Linearity can be inferred on the basis of the principle of superposition and from bootstrap experiments which span 16 or more decades of flux. Indeed, such experiments have been performed with a scintillation detector. Corrections had to be made for slow signal components in the plastic scintillator, which are observable in the steady state but not when measuring intense transient radiation fluxes. By comparison with other detectors under identical high- and low-flux conditions, the linearity of the other detectors is also assured. In this regard, the detectors should also be relatively noise free so that calibrations are accurate.

\section{Speed}

The diagnostic system must be significantly faster than the short-duration processes being investigated to achieve good time resolution. Thus, intrinsically fast phenomena such as generation and collection of induced charge, Cerenkov light, or scintillations must be applied to detect neutrons, gamma rays, or $x$ rays. In addition, the sensitive volume of the detector should be configured to minimize time dispersion. Although there are some applications of optical-fiber waveguides, the signal link is generally a coaxial cable. This typically has foam dielectric $(\mathrm{v} / \mathrm{c}=0.9)$ and $50 \Omega$ impedance. Coaxial cable introduces substantial signal dispersion which can be reduced with a high-pass filter circuit or equalizer that flattens the cable's frequency response. Even so, the detector usually has a faster time response than the coaxial cable signal link. Electrical signals are recorded with wide-bandwidth, unamplified oscilloscopes in which the signal voltage is applied directly to the vertical deflection plate of the oscilloscope cathode ray tube (CRT).

Accurate time measurement is also important. Time information can be accurately carried with the signal being recorded by superimposing a small sinusoidal voltage of appropriate frequency on the CRT horizontal sweep voltage, as shown in Fig. 4. This presentation is called a "Rossi" after Bruno Rossi who, with Robert Wilson, performed prompt diagnostics of the first nuclear explosive. Naturally, all oscilloscopes are accurately crosstimed. Recording of high-speed optical signals transmitted by optical-fiber waveguides is accomplished with an electronic streak camera that can simultaneously record output from many optical fibers.

\section{Insensitivity}

The flux in a nuclear explosion is unusually high. In many instances, the unamplified detector signals can be too large for the available recorders and must be attenuated. The intrinsically fast phenomena applied to detection have cross sections* such that detector sensitivity depends strongly on the number of atoms in the radiation beam (in general, on detector volume for neutrons and gamma rays and on area for $x$ rays). The number of atoms can be controlled through either the area of the collimated beam or the thickness of the detector. If the detection scheme relies on collection of secondary particles, the solid angle ${ }^{\dagger}$ subtended by the collector can be varied. Alternatively, the flux in the beam can be reduced by placing $a b$ sorbers between the source and the detector or by measuring the flux scattered by a known scatterer. Indeed, methods that are not possible in the laboratory can be very useful in nuclear-explosive diagnostics because the flux is so high.

\section{Wide Dynamic Range}

Frequently, it is necessary to measure the gamma-ray output over 10 or more decades. Clearly, such a gamma-ray measurement cannot be accomplished with a single detector. Similarly, each recorder has a limited dynamic range. Several detectors with different sensitivities, distances, collimation, and beam attenuation must be used, and several recorders with different sensitivities and sweep speeds are used to measure the output of a single detector. In this way, there is high assurance that the signals will be properly recorded, despite uncertainties in the predicted magnitude and shape. Figure 5 shows a typical detector circuit diagram.

\footnotetext{
* Cross section, $\sigma$, expressed as an effective area of the target particle, is the quantitative measure of the probability of interaction Cross sections are frequently expressed in units called barns One barn is equivalent to $10^{24} \mathrm{~cm}^{2}$ projected area For thin targets, the beam is not strongly attenuated and the number of interactions per unit time is given by the expression $N_{r}=\phi N_{T} \sigma A_{T} t$, where $\phi$ is flux, $N_{T}$ is the number of target particles per unit volume, $t$ is the thickness of the target, and $A_{T}$ is the target area If the target is thick, and the beam strongly attenuated, the number of interactions per unit time is given bv $N_{r}=\phi A_{T}\left(1-e^{N_{T} \sigma t}\right)$

${ }^{+}$The solld angle is the projected area of the collector d1vided by the square of the distance between the source and the detector, $\Omega=\mathrm{A} / \mathrm{R}^{2}$ steradians
} 


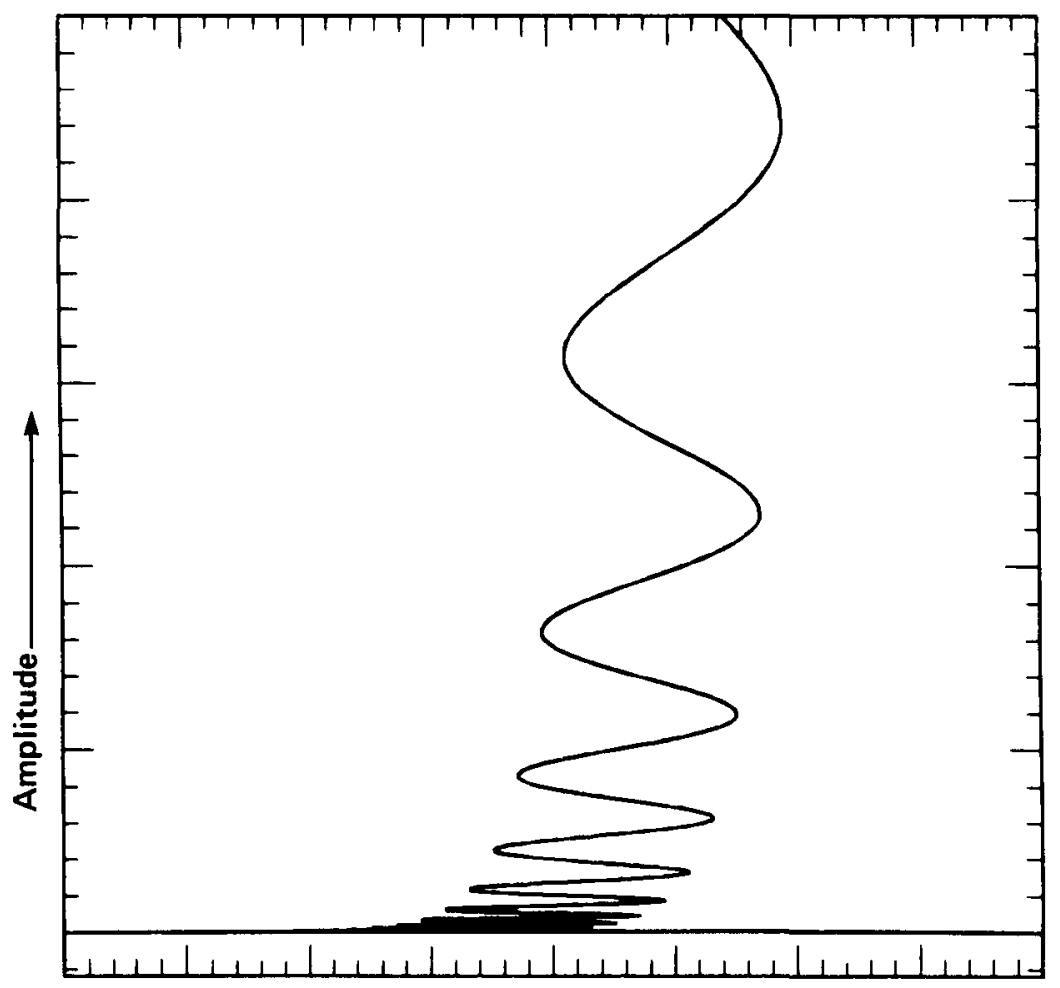

Figure 4. An exponentially rising signal with Rossi oscillations superimposed on the horizontal (time) sweep has accurate time information. This signal has a large Rossi amplitude, and sacrifices accuracy at low signal amplitude for timing accuracy at larger amplitude.

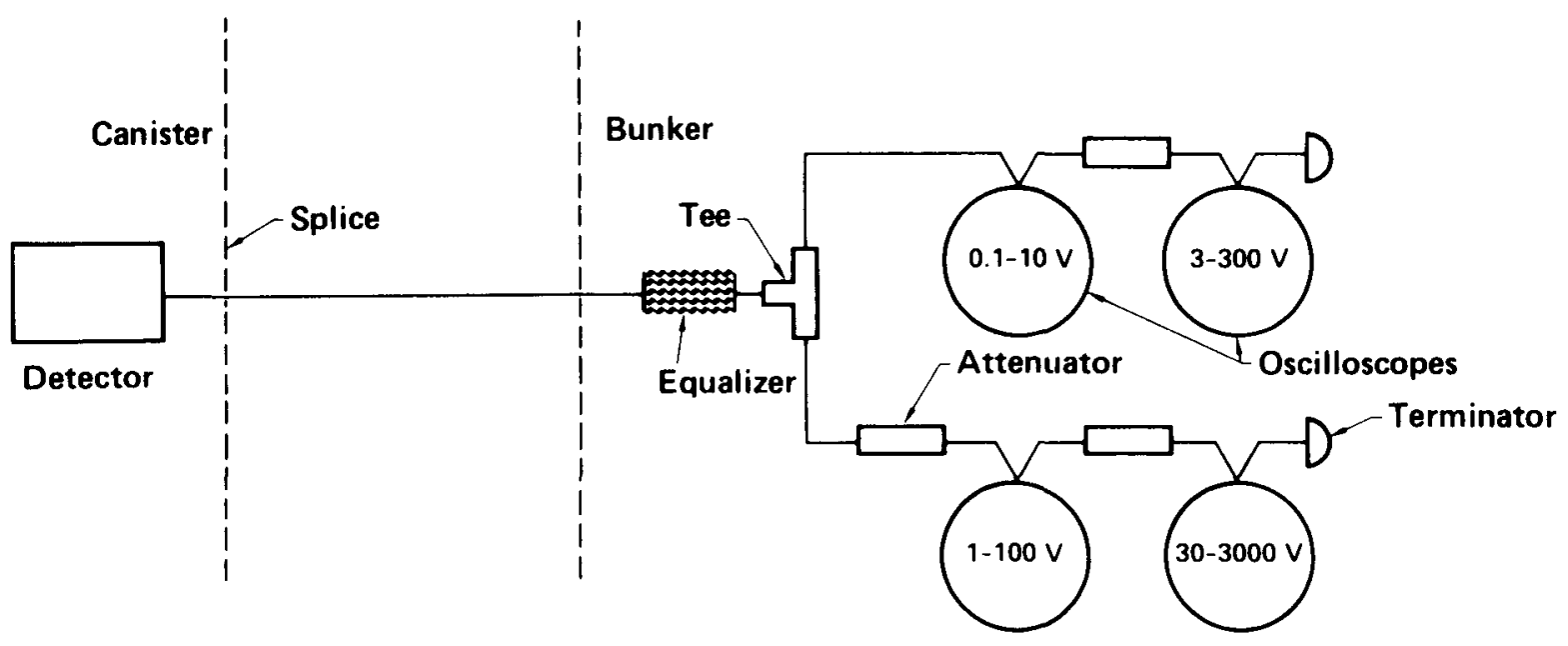

Figure 5. The signal from the detector feeds directly to the vertical deflection plates of a high-speed traveling-wave oscilloscope. The bandwidth is improved because of the equalizer, a lumped RLC (resistance-inductance-capacitance) high-pass filter circuit. The recording dynamic range is $3 \times 10^{4}$. Auxiliary trigger generators (not shown) are necessary to record at the appropriate time. The signals are photographed on high-speed film by cameras that are closed very shortly after the signals arrive. 


\section{Reliability}

The "one chance" nature of the experiment influences all aspects of detector design, fabrication, and testing. Detectors should be rugged, insensitive to temperature variation, and have long- term stability. They are kept as simple as possible to assure reliability. Oscilloscopes are checked frequently for vertical deflection sensitivity, cross timing, and sweep speed to assure stability of the recording system.

\section{Neutron Diagnostics}

\section{Detection of Neutrons}

The free neutron is a product of nuclear reactions. Its mass $\left(m_{n}=1.675 \times 10^{-27} \mathrm{~kg}\right)$ is slightly greater than that of the proton $\left(\mathrm{m}_{\mathrm{p}}=1.672 \times\right.$ $10^{-27} \mathrm{~kg}$ ). Because the neutron has mass, its kinetic energy, $E_{n}$, and velocity, $v$, are related in the nonrelativistic approximation by the expression, $E_{n}=m_{n} v^{2} / 2$. $^{*}$ The neutron is uncharged and is more highly penetrating than gamma rays and $x$ rays, which interact strongly with atomic electrons. It must be detected via nuclear reactions that produce ionization, which is easy to measure. The neutron detectors used are intrinsically fast and are based on the best established neutron cross sections, in particular, elastic scattering of protons and fission of ${ }^{235} \mathrm{U}$ and ${ }^{238} \mathrm{U}$. Although less accurately known, nonelastic scattering is also useful because easily detectable gamma rays are produced virtually simultaneously in nonelastic scattering events.

Neutron detection requires formation of a well-defined neutron beam. This is accomplished as shown in Fig. 6. Both the source and the detector are shielded and collimated. An anti-scattering collimator is positioned roughly midway between the two defining collimators to reduce both the direct and the scattered neutron fluxes which illuminate the collimator that is closest to the detector. This reduces the background significantly. With this kind of beam formation, both the source and the detector volumes are well-defined.

\footnotetext{
* For very accurate work, the relatıvistic expression is used Velocity is given by$$
v=c \sqrt{1-\left(\frac{m_{n} c^{2}}{E_{n}+m_{n} c^{2}}\right)^{2}}
$$

where $c$ is the speed of light $\left(2997 \times 10^{8} \mathrm{~m} / \mathrm{s}\right), E_{n}$ is the kinetic energy, and $m_{n} c^{2}$ is the neutron rest energy (9396 MeV) At 14 $\mathrm{MeV}$, the correct relativistic calculation for $\mathrm{v}$ is $11 \%$ smaller than the nonrelativistic calculation
}

\section{Elastic Scattering of Protons}

Because $m_{n} \simeq m_{p}$, elastic scattering of protons can be thought of in terms of billiard ball collisions. For fast neutrons, a good approximation to the total scattering cross section is $\sigma_{\mathrm{T}}=4.5$ $E_{n}{ }^{-07}$ barns, where $E_{n}$ is in MeV. The energy acquired by the proton is $E_{p}=E_{n} \cos ^{2} \theta$, where $\theta$ is the angle which the proton is scattered relative to the incident neutron direction (taken as $0^{\circ}$ ). All proton energies between 0 and $E_{n}$ are equally probable. Because of this, it can be shown that $\mathrm{d} \sigma / \mathrm{d} \Omega$, the probability of scattering the proton per unit solid angle, $\mathrm{d} \Omega$, at polar angle, $\theta$, (differential scattering cross section) is

$\frac{\mathrm{d} \sigma}{\mathrm{d} \Omega}=\frac{\sigma_{\mathrm{T}}}{\pi} \cos \theta$

Thus, it is advantageous to detect protons at relatively small angles because the differential cross section and the recoil proton energy are higher.

A recoil proton detector is shown in Fig. 7 . The protons are in a thin polyethylene target. Since the protons are charged, they interact very strongly with matter. Therefore, the polyethylene should be thin so that there is a high probability of proton escape without a large energy loss or large angular deflection from Coulomb scattering. The proton detector is set at an angle of $30^{\circ}$. The recoil proton energy is $10.5 \mathrm{MeV}$. To obtain high sensitivity, a silicon ( $\mathrm{Si}$ ) detector is operated as a high-current source. When operated with a highbias voltage, a Si PIN detector can deliver linear output current of approximately 3 to $10 \mathrm{~A}$ with a 3 to $5 \mathrm{~ns}$ response time. Each proton generates $\sim 10^{6}$ electrons in the Si. A Faraday cup, which measures the equivalent of 1 electron per proton, is used when very low sensitivity is required. Electrons and lower-energy protons scattered toward the detector are suppressed by an aluminum absorber and a weak magnetic field between the polyethylene and the detector. To operate properly, the entire assembly is evacuated. Care must 

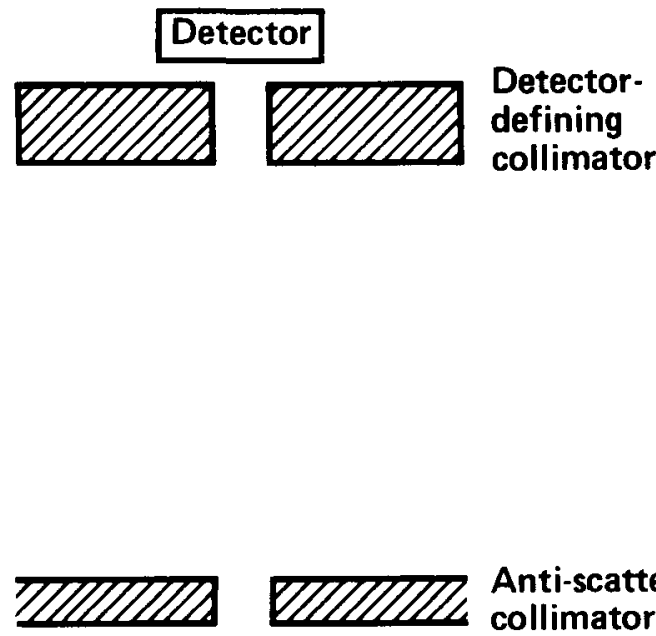

Anti-scattering collimator
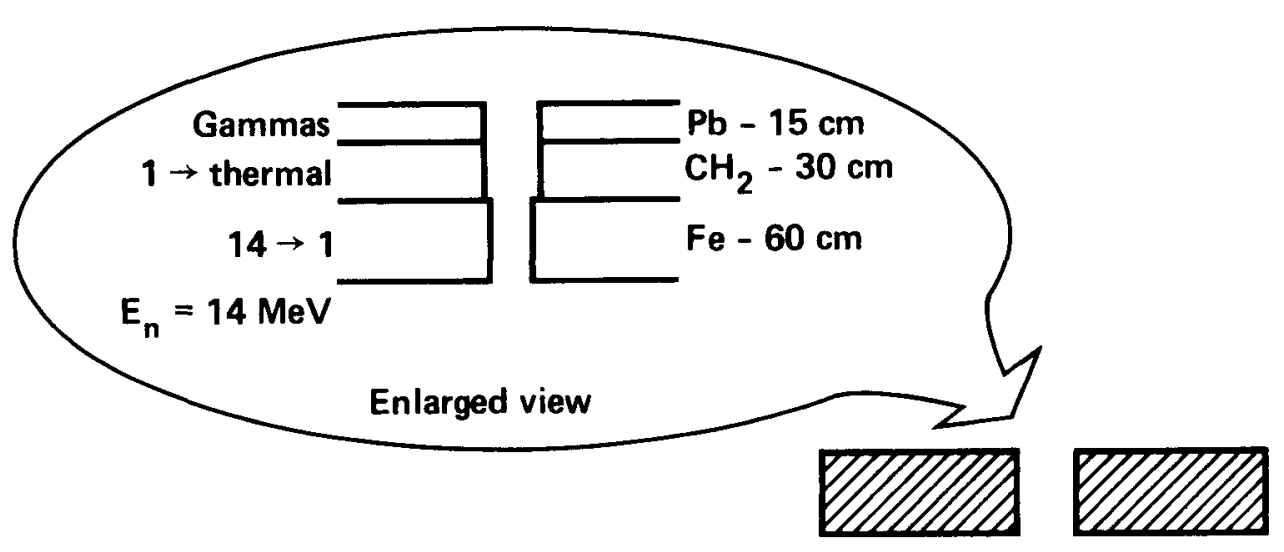

Source-

defining

collimator

\section{O Source}

Figure 6. Schematic diagram of neutron collimation (not to scale). The source collimator is generally larger than the source and is several neutron mean-free-paths thick, as shown in the enlarged view. Thermonuclear (14-MeV) neutrons lose energy in iron (Fe) mainly by nonelastic scattering. The lower-energy neutrons are thermalized and captured or absorbed by hydrogen in the polyethylene $\left(\mathrm{CH}_{2}\right)$. The lead is added to absorb gamma rays arising from neutron capture. The detector defining collimator is similarly constructed. An anti-scattering collimator, ideally located midway between the source and the detector, reduces the number of neutrons that shine on the detector collimator and also restricts the detector's view of the source collimator. Its diameter is larger than the detector-collimator diameter.

be taken to maintain the temperature of the $\mathrm{Si}$ within its operating range. When the background is unusually large or uncertain, magnetic spectrometry is helpful. This allows neutron energy to be measured redundantly, both by time of flight and with the magnetic spectrometer. It is also possible to add more shielding around the detector with this technique. An auxiliary detector located out of the recoil proton beam is used to record background from extraneous sources.

\section{Fission of Uranium}

In fissioning, ${ }^{235} \mathrm{U}$ and ${ }^{238} \mathrm{U}$ release approximately $200 \mathrm{MeV}$. Most of this energy is carried by fission fragments. ${ }^{235} \mathrm{U}$ has an appreciable fission cross section for thermal neutrons, while ${ }^{238} \mathrm{U}$ has 

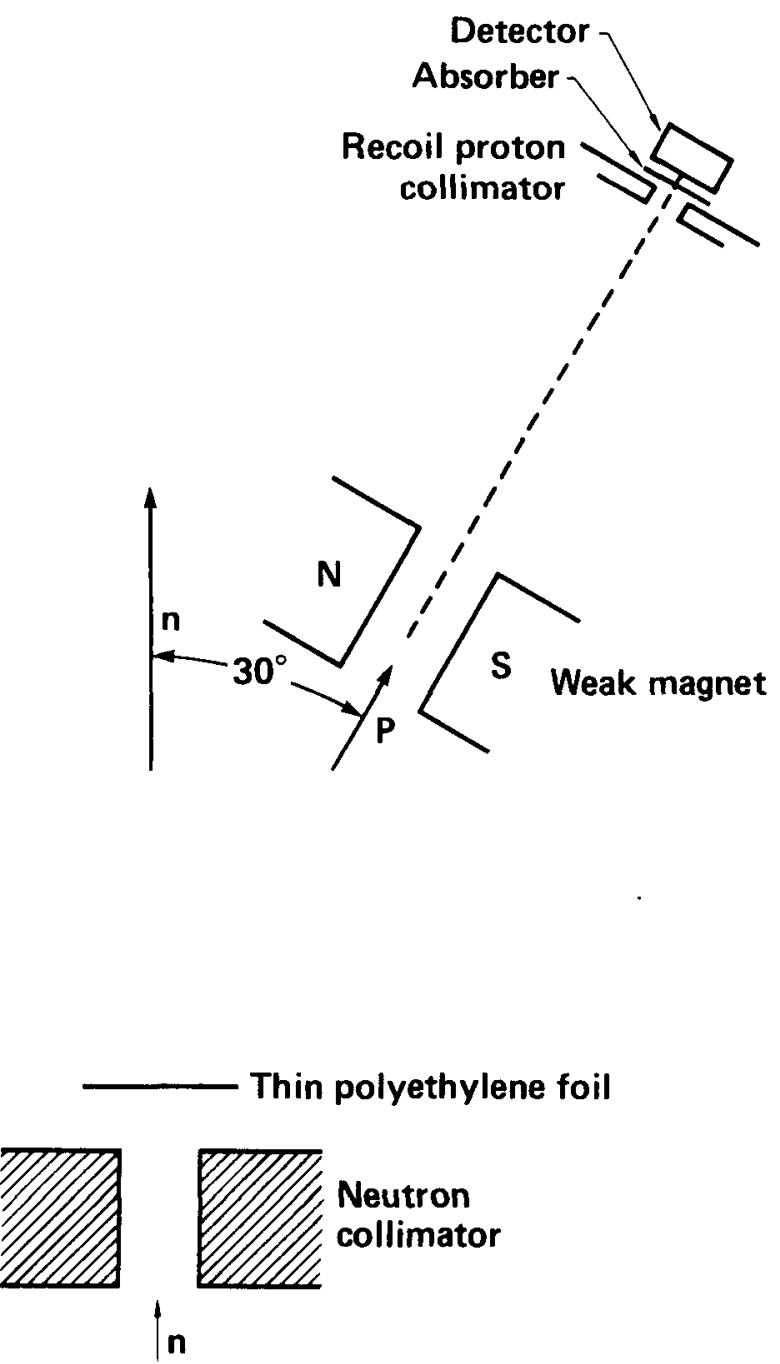

Figure 7. Schematic diagram of a recoil proton detector. The collimated neutrons collide elastically with protons in the polyethylene. Protons scattered at $30^{\circ}$ are detected with a silicon (Si) detector that is shielded from light and lowenergy charged particles by a very thin absorber and a weak magnetic field, respectively.

a fission threshold of approximately $1.5 \mathrm{MeV}$. This threshold allows some discrimination against neutron scattering. Detectors which rely on fission fall in two categories, those which derive their signal from the fission fragments and those which derive their signal from the prompt gamma rays produced in fission.

Fission Fragment Detection. Fission fragments are relatively massive, are highly ionizing, and, therefore, have very short range. To have a high probability of detection, the fission target must be thin enough for fission fragments to escape. Fission-fragment energy is detected with a nearby $\mathrm{Si}$ detector operated in the current mode. Each fission fragment has approximately $80 \mathrm{MeV}$ of energy and will generate approximately $3 \times$ $10^{7}$ electrons in the $\mathrm{Si}$ detector. If a higher-speed, less-sensitive detector is required, a fission foil detector such as the one shown in Fig. 8 might be used. In this detector, there are two very thin layers of ${ }^{235} \mathrm{U}_{3} \mathrm{O}_{8}$ on both sides of a beryllium-foil substrate. Each escaping fission fragment induces the emission of approximately 300 low-energy secondary electrons which are collected by thin, biased beryllium anodes. Since the space between the electrodes is quite small, and the bias voltage high, this detector is fast $(\sim 1 \mathrm{~ns})$. The fission foil detector can deliver over $20 \mathrm{~A}$ of linear output current. Owing to the thickness and low atomic number of the Be electrodes, this detector has very low sensitivity to gamma rays.

Fission Gamma-Ray Detection. Each fission produces approximately $8 \mathrm{MeV}$ of prompt

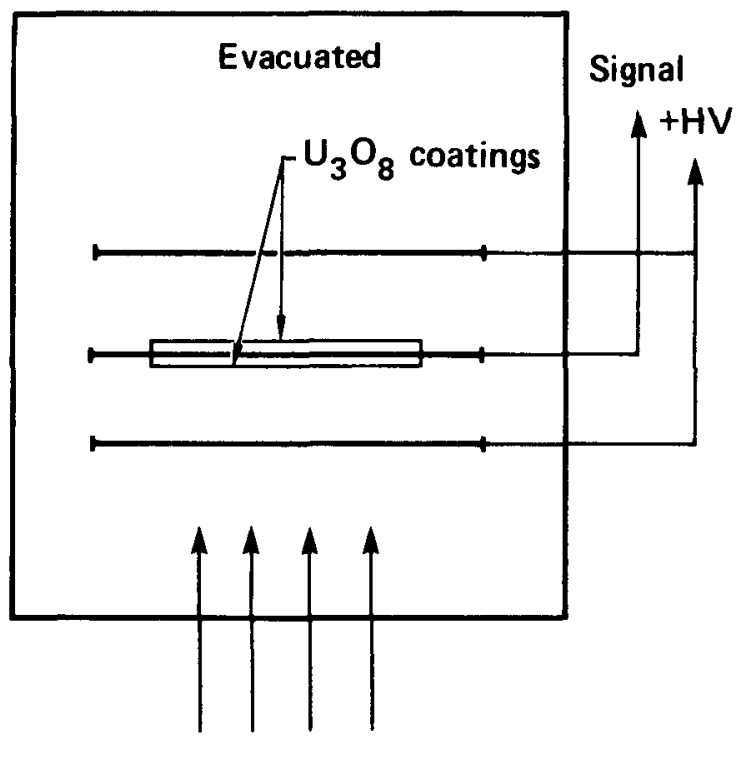

Neutrons

Figure 8. Schematic diagram of a fission foil neutron detector. Very thin layers of ${ }^{235} \mathrm{U}_{3} \mathrm{O}_{8}$ are applied on both sides of the Be anode. Each escaping fission fragment generates approximately 300 low-energy secondary electrons, which are collected by thin Be anodes. The sensitivity to gamma rays is small because the detector elements are very thin. The anodes are biased to assure efficient electron collection. 
gamma-ray energy. This effect is applied in detecting neutrons with a detector such as that shown in Fig. 9. The fission gamma rays from a $U$ disk clad with aluminum generate a large current of Compton electrons, which is recorded as a positive signal. This detector has $\sim 1$ ns response time and can deliver over $100 \mathrm{~A}$ of linear output current. If the thickness of the $U$ disk is properly chosen, the external (from the nuclear explosion) gamma-ray-induced electron currents between the uranium-aluminum cathode and ground will nearly cancel.

\section{Nonelastic Scattering}

In nonelastic scattering, the target nucleus is left in an excited state which promptly decays with the emission of gamma rays; the gamma rays generate a current of Compton electrons in a detector similar to the fission gamma-ray detector. Alternatively, the nonelastic gamma rays produced in a converter can be detected with a gamma-ray detector located safely outside the neutron beam.

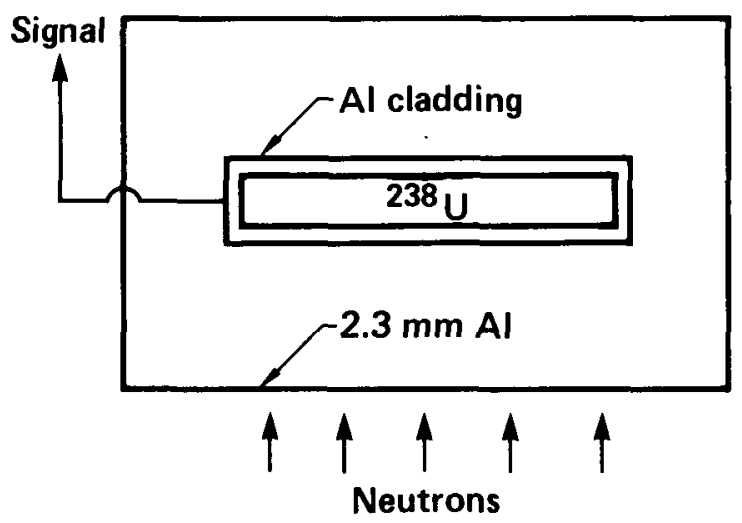

Figure 9. Schematic diagram of a fission gamma-ray detector. Prompt fission gamma rays produced in the $U$ part of the cathode generate a current of Compton electrons from the aluminum cladding to ground. Sensitivity to external fission gamma rays from the device is minimized by choosing the thickness of the $U$ so that the Compton current from the entrance window to the cathode cancels the Compton current from the cathode to the exit window. If ${ }^{238} U$ is used, the detector has a threshold at $E_{n}=1.5 \mathrm{MeV}$. This detector requires no bias voltage to operate properly.

\section{Time-Dependent Output Measurements}

Of major diagnostic interest is the neutron production rate since this is proportional to the fission rate, or for a thermonuclear explosive, the fusion rate. Because the neutron has mass, its velocity is determined by its energy, that is,

$\mathrm{v}=\sqrt{\frac{2 \mathrm{E}}{\mathrm{m}_{\mathrm{n}}}}$.

If there is a source energy spread, $\Delta \mathrm{E}$, there will be a velocity spread, $\Delta v$, and a time spread, $\Delta t$, at the detector given by

$\Delta \mathrm{v}=\frac{\mathrm{v} \Delta \mathrm{E}}{2 \mathrm{E}}$,

and

$\Delta \mathrm{t}=\frac{\mathrm{t} \Delta \mathrm{E}}{2 \mathrm{E}}$,

where $t$ is the time of flight.

The fission neutron spectrum is very broad and continuous, extending to above $10 \mathrm{MeV}$, with an average energy of approximately $2 \mathrm{MeV}$. Thermonuclear neutrons are produced in the predominant reaction

$\mathrm{D}+\mathrm{T} \rightarrow \mathrm{n}+{ }^{4} \mathrm{He}+17.6 \mathrm{MeV} \quad .^{*}$

The neutron energy would be approximately $14 \mathrm{MeV}(4 / 5 \times 17.6 \mathrm{MeV}$, from the laws of conservation of energy and momentum) if the particles could react with zero energy. Clearly this is not the case, because high temperature is required for the reaction to proceed. The rate at which thermonuclear reactions take place, $\dot{\mathrm{N}}_{\mathrm{t} n}$, is given by

$\dot{\mathrm{N}}_{\mathrm{tn}}=\mathrm{N}_{\mathrm{D}} \mathrm{N}_{\mathrm{T}} \overline{\sigma \mathrm{v} V}$

where $N_{D}$ and $N_{T}$ are the deuteron and triton atom densities respectively, $\overline{\sigma \mathrm{v}}$ is the Maxwellaveraged reaction-rate constant, and $\mathrm{V}$ is the reaction volume. Figure 10 shows how rapidly $\overline{\sigma v}$ increases with increasing temperature. Most thermonuclear neutrons are produced in high-velocity

\footnotetext{
* The reaction $\mathrm{D}+\mathrm{D} \rightarrow \mathrm{n}+{ }^{3} \mathrm{He}+3.2 \mathrm{MeV}$ produces $2.5-\mathrm{MeV}$ neutrons but at a much lower rate.
} 


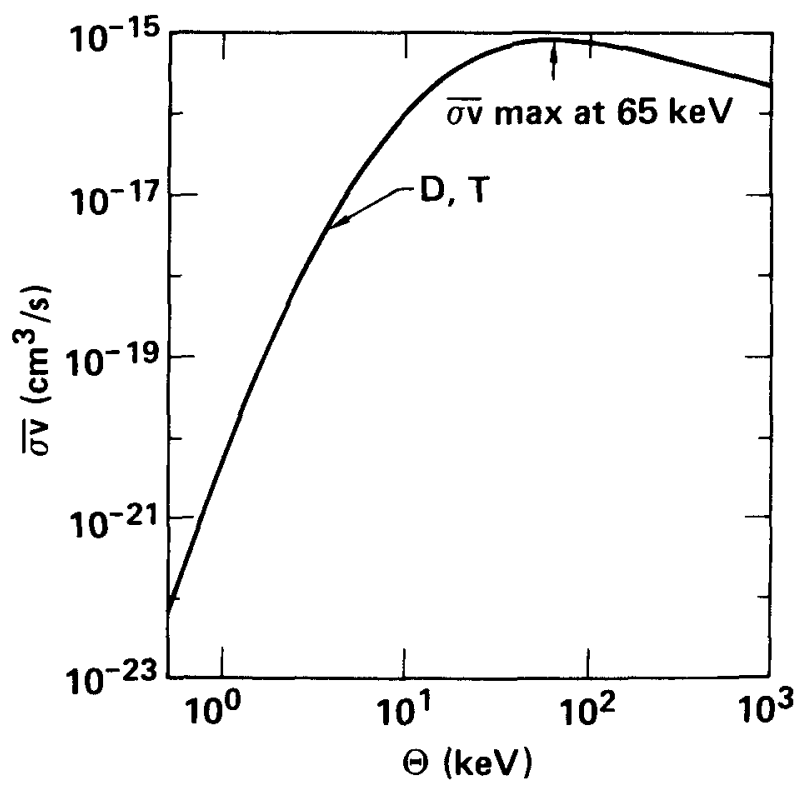

Figure 10. A $\overline{\sigma v}$ curve for the $D, T$ reaction. The Maxwell-averaged $D, T$ reaction-rate constant increases very rapidly with increasing temperature. As a result, the thermonuclear neutrons have a large energy spread.

reactions. This tends to broaden the neutronenergy spread and adversely affects time resolution. The energy spread in $\mathrm{MeV}$ is given by the expression

$\Delta \mathrm{E}_{\mathrm{n}} \simeq 0.178 \mathrm{v} \Theta$

where $\Theta$ is the particle temperature in $\mathrm{keV}$. The corresponding time spread at a detector $\mathrm{R}$ meters from the source is

$\Delta t=0.112 \mathrm{R} \sqrt{ } \Theta$

where $\Delta t$ is expressed in nanoseconds.

From these considerations, we see that because of time-of-flight dispersion, the neutron detection rate is lower than the production rate and that $\Delta \mathrm{t}_{\mathrm{s}}$, the signal time-width, has three main contributions, namely $\Delta t_{r^{\prime}}$ the reaction time, $\Delta t_{\theta^{\prime}}$ the time-of-flight spread, and $\Delta t_{d}$, the detector response time, with $\Delta \mathrm{t}_{\theta}$ generally the major contributor. This can be expressed as

$\Delta \mathrm{t}_{\mathrm{s}}=\sqrt{\left(\Delta \mathrm{t}_{\mathrm{r}}\right)^{2}+\left(\Delta \mathrm{t}_{\theta}\right)^{2}+\left(\Delta \mathrm{t}_{\mathrm{d}}\right)^{2}}$.

As a result, remote detectors give neutron-energy spectra.
However, there is a method of obtaining the thermonuclear energy production rate from a neutron measurement. This is illustrated in Fig. 11. The relatively undispersed $14-\mathrm{MeV}$ neutrons are nonelastically scattered in a converter and produce prompt gamma rays from very short-lived excited nuclear states. These are detected with a high-speed gamma-ray detector that has a relatively flat energy response. The converter is thin and is configured to minimize the spread in gamma-ray arrival time at the detector. A spherical shell with its center rotated an angle $\beta=\mathrm{v} / \mathrm{c}$ ( $v$ is the speed of $14-\mathrm{MeV}$ neutrons and $c$ is the speed of light) above the center of the neutron source is an excellent approximation of the optimal shape. Discrimination against fission neutrons is accomplished by the choice of converter material, which should have a large nonelastic scattering cross section, high-energy gamma rays and a high-energy neutron reaction threshold. A good choice is ${ }^{16} \mathrm{O}$ in $\mathrm{BeO}$. Further discrimination against gamma rays from the device is accomplished by locating the detector at an angle of $90^{\circ}$ or greater from the neutron direction. As will be seen later, photons scattered at $90^{\circ}$ have energy below $0.51 \mathrm{MeV}$ and are preferentially absorbed in a lead filter between the converter and the gamma-ray detector. Other methods of obtaining fission and fusion reaction rates will be discussed in the section on gamma-ray measurements.

\section{Spectrometry}

The time-of-flight spectrum is obtained with a remote detector. The rate at which neutrons pass a detector of area, $A_{D}$, at a distance, $R$, from a pulsed neutron source is given by

$\frac{\mathrm{dn}}{\mathrm{dt}}=C E^{3 / 2} m_{n}{ }^{-1 / 2} \frac{A_{D}}{R^{3}} \frac{d n}{d E}$ ，

where $\mathrm{dn} / \mathrm{dE}$ is the source energy spectrum and $\mathrm{C}$ is a constant of proportionality. The current, $i$, generated in a detector viewing the neutron source is given by $\mathrm{i}=\mathrm{ST} \cdot \mathrm{dn} / \mathrm{dt}$, where $\mathrm{S}$ is the detector sensitivity, expressed in units of $\mathrm{C} / \mathrm{n}$, and $\mathrm{T}$ is the transmittance of materials placed in the neutron beam. From these expressions we conclude that the neutron beam must be well defined and that the source and detector must be well shielded to minimize detection of scattered neutrons. This is particularly important because the signal is heavily weighted in favor of the more energetic neutrons. In time-of-flight spectrometry, 

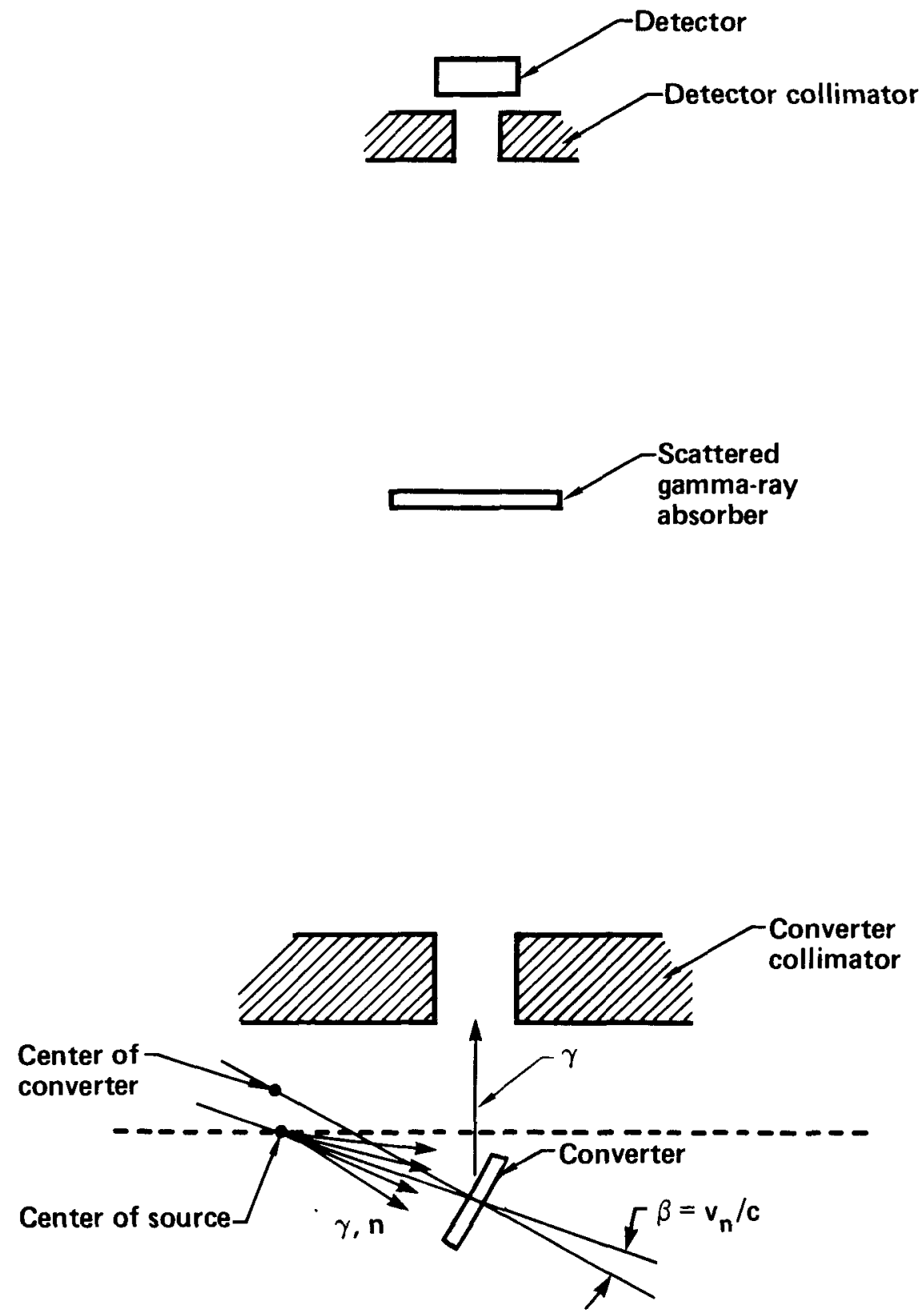

Figure 11. The fusion neutron production rate is proportional to the nonelastic gamma-ray production rate in a spherical converter located very close to the device, where time-of-flight dispersion is negligible. The gamma-ray detector is located at an angle of $90^{\circ}$ or more to discriminate against gamma rays produced in the exploding device. By inclining the converter at an angle of $0.17 \mathrm{rad}$, the neutron-induced gamma-ray signals from all elements of the converter arrive at the detector simultaneously.

energy resolution improves with increasing flight time according to $\Delta \mathrm{E}=2 \mathrm{E} \Delta \mathrm{t} / \mathrm{t}$.

All of the detectors mentioned previously are useful in time-of-flight measurements. It should be pointed out that magnetic spectrometry of recoil protons is particularly valuable when the flux of scattered neutrons is high. 


\section{Gamma-Ray Diagnostics}

\section{Detection of Gamma Rays}

Gamma rays are electromagnetic radiation or photons which arise from radiative decay of excited nuclear states. In the context of nuclearexplosive diagnostics, gamma rays have energy above $0.1 \mathrm{MeV}$. Gamma rays travel at the speed of light and have frequency $v=\mathrm{E}_{\gamma} / \mathrm{h}$, where $\mathrm{E}_{\gamma}$ is the transition energy and $h$ is Planck's constant $\left(\mathrm{h}=6.67 \times 10^{-34} \mathrm{~J} \cdot \mathrm{s}=4.16 \times 10^{-21} \mathrm{MeV} \mathrm{s}\right)$. The photon has momentum $\mathrm{p}_{\gamma}=\mathrm{h} v / \mathrm{c}=\mathrm{E}_{\gamma} / \mathrm{c}$. While each nuclear transition gives a discrete energy, there are so many different excited nuclear species produced in nuclear fission that the gamma-ray spectrum is smooth. Because of its electromagnetic nature, the photon interacts strongly with charge or its electric field. Table 1 lists the interactions which are most important in nuclear-explosive diagnostics. The interactions are independent of each other, and hence the total cross section or interaction probability can be found by summing the cross sections of all interactions. Thus, $\sigma_{\text {TOTAL }}=\sigma_{\mathrm{PE}}+\sigma_{\mathrm{SCATT}}+\sigma_{\mathrm{PP}}$. Cross sections for effects which are important in gamma-ray and $x$-ray diagnostics are also among the best established in physics. Figure 12 shows for aluminum and lead how these interactions depend on energy.

Except in the case of elastic scattering, interactions with atoms result in the emission of electrons which are easy to detect. In the photoeffect which dominates at low photon energy, the photon is absorbed and a tightly bound inner shell electron is emitted. The electron has kinetic energy

$\mathrm{E}_{\mathrm{e}}=\mathrm{E}_{\gamma}-\mathrm{B}_{\mathrm{e}}$, where $B_{e}$ is the electron-binding energy. Virtually simultaneous with electron emission is the emission of fluorescence $x$ rays as the atom's innershell vacancy is filled. Photoelectric cross sections exhibit a large increase at the binding energy of each atomic shell. This atomic property can be exploited in x-ray spectrometry. At low energy, the photon has little momentum and the electrons are preferentially emitted transverse to the direction of the incident photon, that is, in the direction of the photon's electric vector. At high energy, where photon momentum is appreciable, photoelectron emission peaks in the forward direction. In elastic or coherent scattering, the photon loses no energy, but its direction is changed. This interaction is important at low energy and at very small scattering angles and can be useful in reducing the gamma-ray or $x$-ray flux to a measurable level. In inelastic or Compton scattering, the photon loses energy to a loosely bound atomic electron with a corresponding change in direction. The energy, $\mathrm{E}_{\gamma}$, of the scattered photon is given by

$E_{\gamma}^{\prime}=\frac{E_{\gamma}}{1+\frac{E_{\gamma}}{m_{e} c^{2}}(1-\cos \theta)}$,

where $m_{e} c^{2}$ is the electron rest energy $(0.511 \mathrm{MeV})$ and $\theta$ is the direction of the scattered photon. For small scattering angles and at low photon energy, very little energy is lost in Compton scattering. Note that photons scattered at $90^{\circ}$ will always have energy below $0.511 \mathrm{MeV}$, and that photons scattered at $180^{\circ}$ (back to the source) will always

Table 1. Interactions of photons with matter.

\begin{tabular}{|c|c|c|c|c|}
\hline \multirow[b]{2}{*}{ Process } & \multirow{2}{*}{$\begin{array}{l}\text { Interaction } \\
\text { center }\end{array}$} & \multirow[b]{2}{*}{ Energy range } & \multicolumn{2}{|c|}{ Variation with } \\
\hline & & & $\bar{Z}$ & $E_{x}, E_{\gamma}$ \\
\hline Photoelectric effect & $\begin{array}{l}\text { Tightly bound } \\
\text { electron }\end{array}$ & $\begin{array}{l}0.1 \mathrm{keV}-500 \mathrm{keV} \\
\text { dominates at } 50 \mathrm{keV}\end{array}$ & $Z^{5}$ & $E^{-3}$ \\
\hline $\begin{array}{l}\text { Coherent or } \\
\text { elastic scattering }\end{array}$ & Bound electrons & $<10 \mathrm{keV}$ & $Z^{2}$ & $\begin{array}{c}\text { None } \\
10 \mathrm{keV}\end{array}$ \\
\hline $\begin{array}{l}\text { Incoherent or } \\
\text { Compton scattering }\end{array}$ & "Free" electrons & $\begin{array}{l}500-1500 \mathrm{keV} \\
\text { dominates at } 1000 \mathrm{keV}\end{array}$ & $\mathrm{Z}$ & $E^{-1}$ \\
\hline Pair production & $\begin{array}{l}\text { Nuclear electric } \\
\text { field }\end{array}$ & $\begin{array}{l}>1022 \mathrm{keV} \\
\text { dominates at } 5000-10000 \mathrm{keV}\end{array}$ & $\mathrm{Z}^{2}$ & $(E-1022)^{2}$ \\
\hline
\end{tabular}




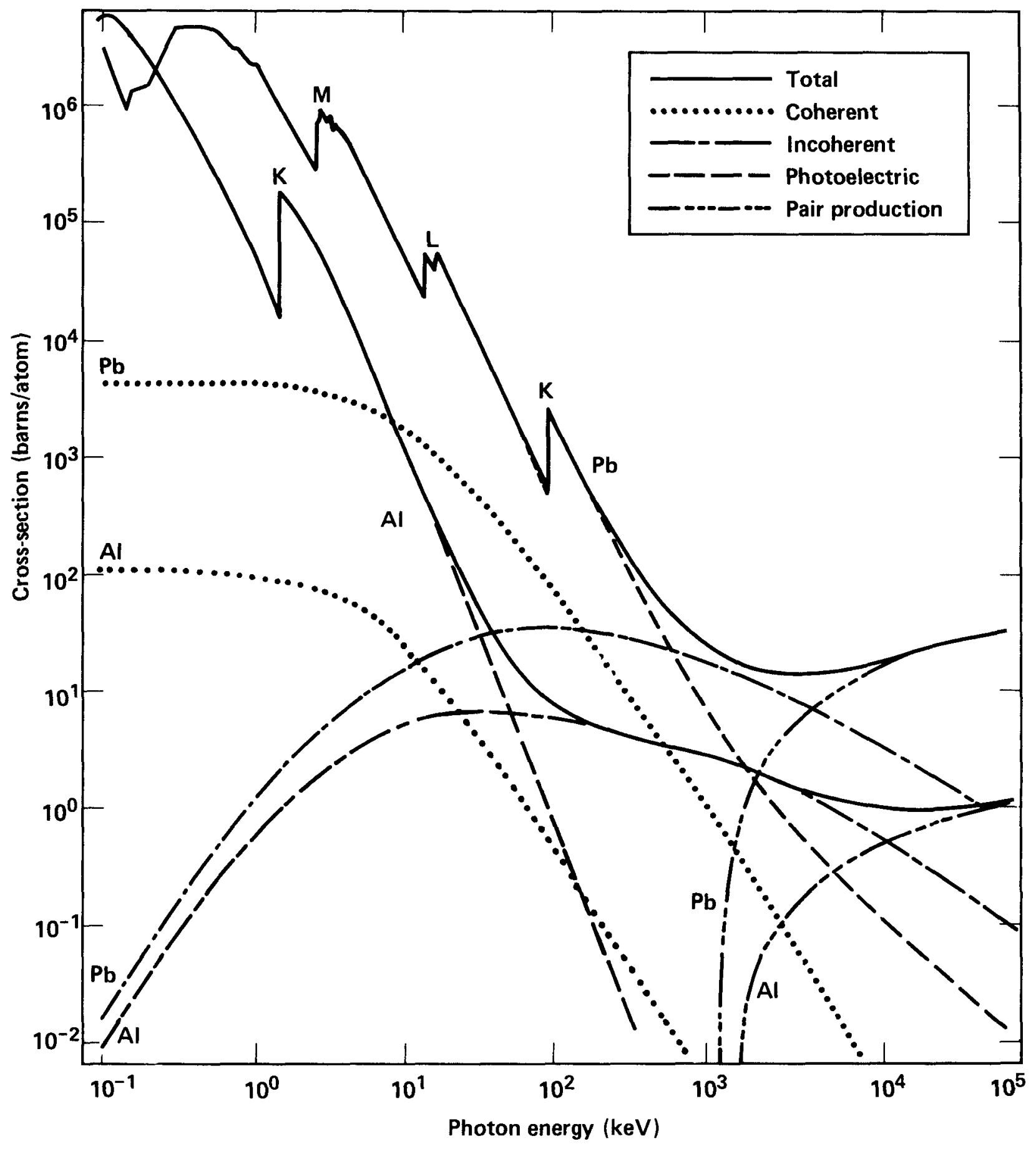

Figure 12. Photon interaction cross sections of aluminum (Al) and lead ( $\mathrm{Pb}$ ) from $100 \mathrm{eV}$ to $100 \mathrm{MeV}$. The sharp increases in photoelectric cross section occur at energies corresponding to the binding energies of the atomic shells. At low energy, the photoelectric absorption dominates. Near $1 \mathrm{MeV}$, Compton or nonelastic scattering is dominant, while at very high energy, pair production has the highest interaction probability. The photon mean-free-path is the reciprocal of the cross section times the atomic density of the element. 
have energy below $0.255 \mathrm{MeV}$. The Compton defect or scattered electron energy is given by

$E_{e}=E_{\gamma}-E_{\gamma}^{\prime}$

The differential scattering cross sections or angular distributions of the scattered photons and electrons are given by the well-known Klein-Nishina formulae. For $E_{\gamma} / m_{e} c^{2}>1$, electrons are scattered predominantly in the forward direction.

Interaction with the nucleus results in emission of photonucleons (neutrons or protons). For most nucleii, the photonuclear emission threshold is about $10 \mathrm{MeV}$. Gamma-ray spectra can be obtained by measuring the flight time of photoneutrons. This is done with ${ }^{2} \mathrm{H}$ or ${ }^{9} \mathrm{Be}$ targets, which have unusually low threshold energies of 2.2 and $1.7 \mathrm{MeV}$, respectively.

Interaction with the nuclear electric field gives rise to pair production, that is, annihilation of the photon with the creation of an electron and a positron (anti-electron). The pair production threshold is $1.02 \mathrm{MeV}\left(2 \mathrm{~m}_{\mathrm{e}} \mathrm{c}^{2}\right)$. While energy is conserved in pair production, there is a broad spectrum of positron and electron energies, making this phenomenon difficult to apply in spectrometry.

These intrinsically fast processes mainly produce electrons that travel close to the speed of light, making it possible to obtain very accurate time-dependent measurements. The $x$-ray flux $\left(\mathrm{E}_{\mathrm{x}}<0.1 \mathrm{MeV}\right)$ can be easily eliminated with a 3-mm-thick lead absorber in the beam, and 14$\mathrm{MeV}$ neutrons, which travel at $0.17 \mathrm{c}$, do not interfere with gamma-ray measurements. Thus, it is generally very easy to achieve gamma-ray selectivity. Table 2 lists the properties of gamma-ray detectors that are useful in diagnostics.

The fastest and least sensitive gamma-ray detector is the Compton diode, shown in Fig. 13. It consists of an insulated cathode inside an evacu- ated cylindrical conductor that is grounded. The cathode is connected to ground externally through a fast oscilloscope. The Compton diode is designed to operate in a $10-\mathrm{cm}$ diameter beam. Gamma-ray induced Compton electrons travel very rapidly from the cathode to ground. Net electron transport is optimized by the choice of materials and thicknesses of the cathode and the entrance and exit windows. The conversion efficiency is approximately $5 \times 10^{-3}$ electrons $/ \mathrm{MeV}$. The output current is linear to approximately $10^{2} \mathrm{~A}$. The sensitivity of this type detector is roughly independent of energy. It has a time response of approximately $0.7 \mathrm{~ns}$. While convenient to use, the cylindrical geometry is not the best for very-highfrequency response. Much better time resolution can be obtained by tailoring the detector configuration to appear electrically as a short section of coaxial cable. Figure 14 shows the components of such a detector. The detector has a very thin entrance window and an auxiliary magnetic field to deflect away from the cathode those electrons scattered from the beam defining collimator and entrance window. A wire screen isolates the detector snout from the $50 \Omega$ structure formed by the cathode and vacuum envelope which approximate confocal ellipses. The $0.15 \mathrm{~ns}$ response time measured for this detector is largely determined by the spread in signal transit time across the 5 -cm long cathode.

Higher sensitivity is obtained with detectors incorporating fast plastic scintillators, which measure absorbed energy. The typical scintillation detector is a $15-\mathrm{cm}$ cube, one gamma-ray mean-freepath thick, ${ }^{*}$ optically coupled to a fast planar

\footnotetext{
* Cross section in units of $\mathrm{cm}^{1}$ is obtained by multiplying $\sigma$ (in units of $\mathrm{cm}^{2} /$ atom) by the atomic density (atoms $/ \mathrm{cm}^{3}$ ) The mean-free-path, given by $\lambda_{\mathrm{m}}=1 / \sigma_{\text {TOT }}$, 15 the thickness required to attenuate the flux to $1 / \mathrm{e}$ of 1 ts original value
}

Table 2. Characteristics of gamma-ray detectors.

\begin{tabular}{|c|c|c|c|c|}
\hline Type & $\begin{array}{c}\text { Sensitivity } \\
\text { (electrons/ } \\
\text { MeV) }\end{array}$ & $\begin{array}{l}\text { Response } \\
\text { time } \\
\text { (ns) }\end{array}$ & $\begin{array}{l}\text { Max. flux } \\
{[\mathrm{MeV} /} \\
\left.\left(\mathrm{cm}^{2} \mathrm{~s}\right)\right]\end{array}$ & $\begin{array}{l}\text { Max. } \\
\text { current } \\
\text { (A) }\end{array}$ \\
\hline $\begin{array}{l}\text { Plastic } \\
\text { scintillator }+ \\
\text { photomultiplier }\end{array}$ & $4 \times 10^{7}$ & $5-8$ & $10^{19}$ & $\sim 0.1$ \\
\hline $\begin{array}{l}\text { Plastic } \\
\text { scintillator }+ \\
\text { planar photodiode }\end{array}$ & 40 & 3 & $10^{19}$ & 20 \\
\hline $\begin{array}{l}\text { Cerenkov }+ \\
\text { planar photodiode }\end{array}$ & 0.4 & 1 & $10^{21}$ & 20 \\
\hline Compton diode & $5 \times 10^{3}$ & 1 & $10^{23}$ & 100 \\
\hline
\end{tabular}


photodiode. The absorbed gamma-ray energy is converted to blue light which generates current in the photodiode. The conversion efficiency is approximately 40 electrons $/ \mathrm{MeV}$, and is roughly independent of energy. With photodiode bias of 8 $\mathrm{kV}$, the maximum linear output current is approximately $20 \mathrm{~A}$. A photomultiplier will give even higher sensitivity at the expense of dynamic range. The response time is approximately 3 to 5 ns. A Cerenkov detector, which has no scintillation material in the plastic, is faster but has lower sensitivity, approximately 0.4 electron $/ \mathrm{MeV}$. The gamma-ray energy threshold for production of Cerenkov light in plastic is approximately $0.2 \mathrm{MeV}$.

Sometimes it is convenient to use a much smaller detector. In such cases a $1-\mathrm{cm}$ diameter $x$ $250-\mu \mathrm{m}$ thick Si PIN junction detector is useful. Since the detector is smaller than the photonbeam diameter, it is self-collimating. The sensitivity is roughly $4 \times 10^{2}$ electrons $/ \mathrm{MeV} \cdot \mathrm{cm}^{2}$, and the response time is approximately $3 \mathrm{~ns}$.

\section{Time-Dependent Output Measurements}

The rate of energy production is a very important indicator of performance. This measurement, called reaction history, is the grandfather of all diagnostics. From it, $\tau$, the generation time, is obtained. This gives a space average, $\alpha$, of the neutron concentration. By definition,

$\alpha=\frac{1}{\tau}=\frac{1}{[\mathrm{n}]} \frac{\mathrm{d}[\mathrm{n}]}{\mathrm{dt}}$,

where [n] is the neutron concentration. As was seen earlier, unless very high energy resolution characterizes the neutron measurement, the desired time resolution cannot be obtained. Since it is very difficult to obtain the requisite neutron energy resolution, the total gamma-ray energy flux is measured instead. Thus,

$\alpha_{\gamma}=\frac{1}{\phi_{\gamma}} \frac{\mathrm{d} \phi_{\gamma}}{\mathrm{dt}}$

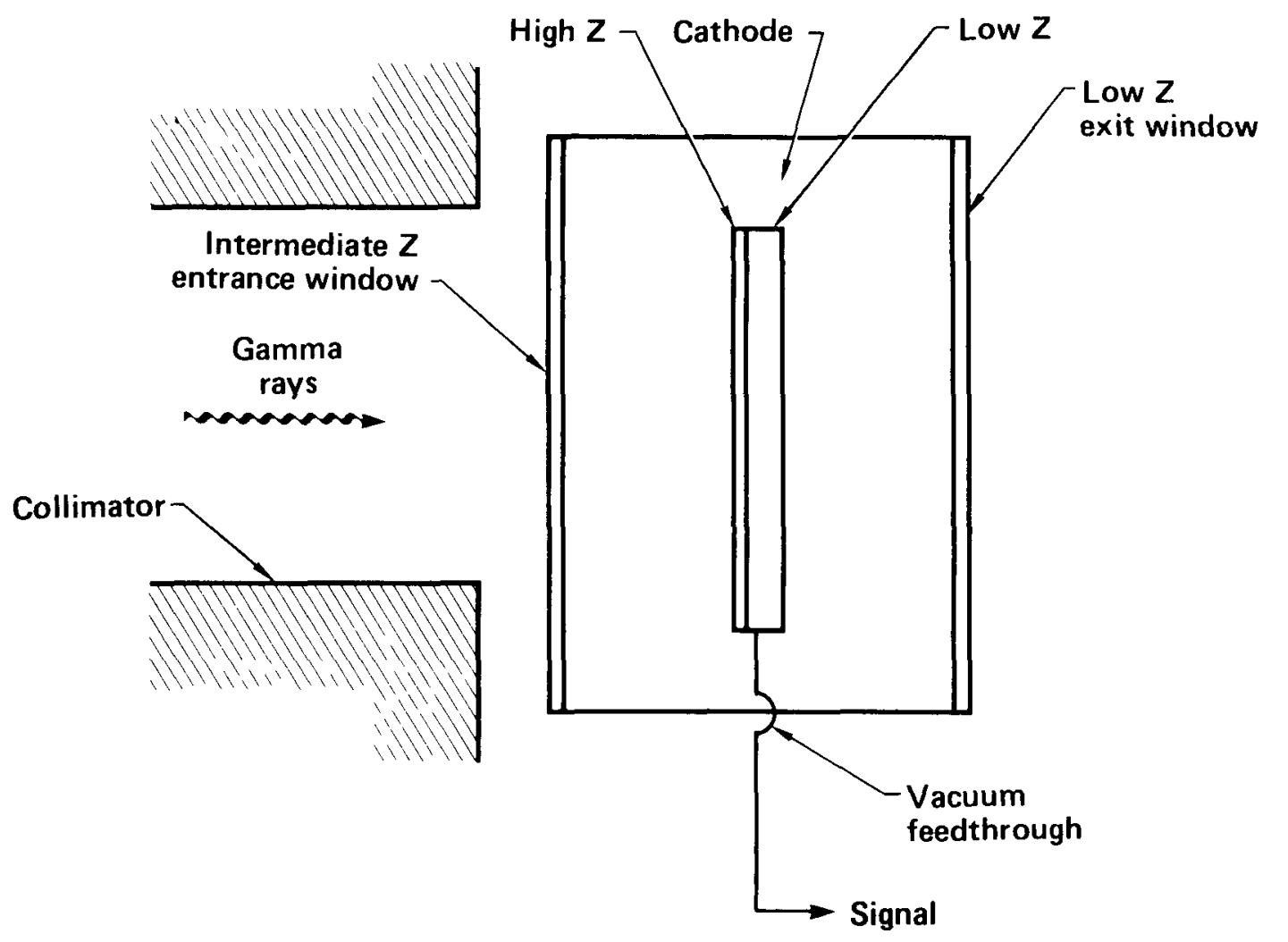

Figure 13. A cylindrical Compton diode whose material and thickness are chosen to optimize sensitivity in the $1-$ to $3-\mathrm{MeV}$ energy range. This detector is designed to operate in a collimated gamma-ray beam. 


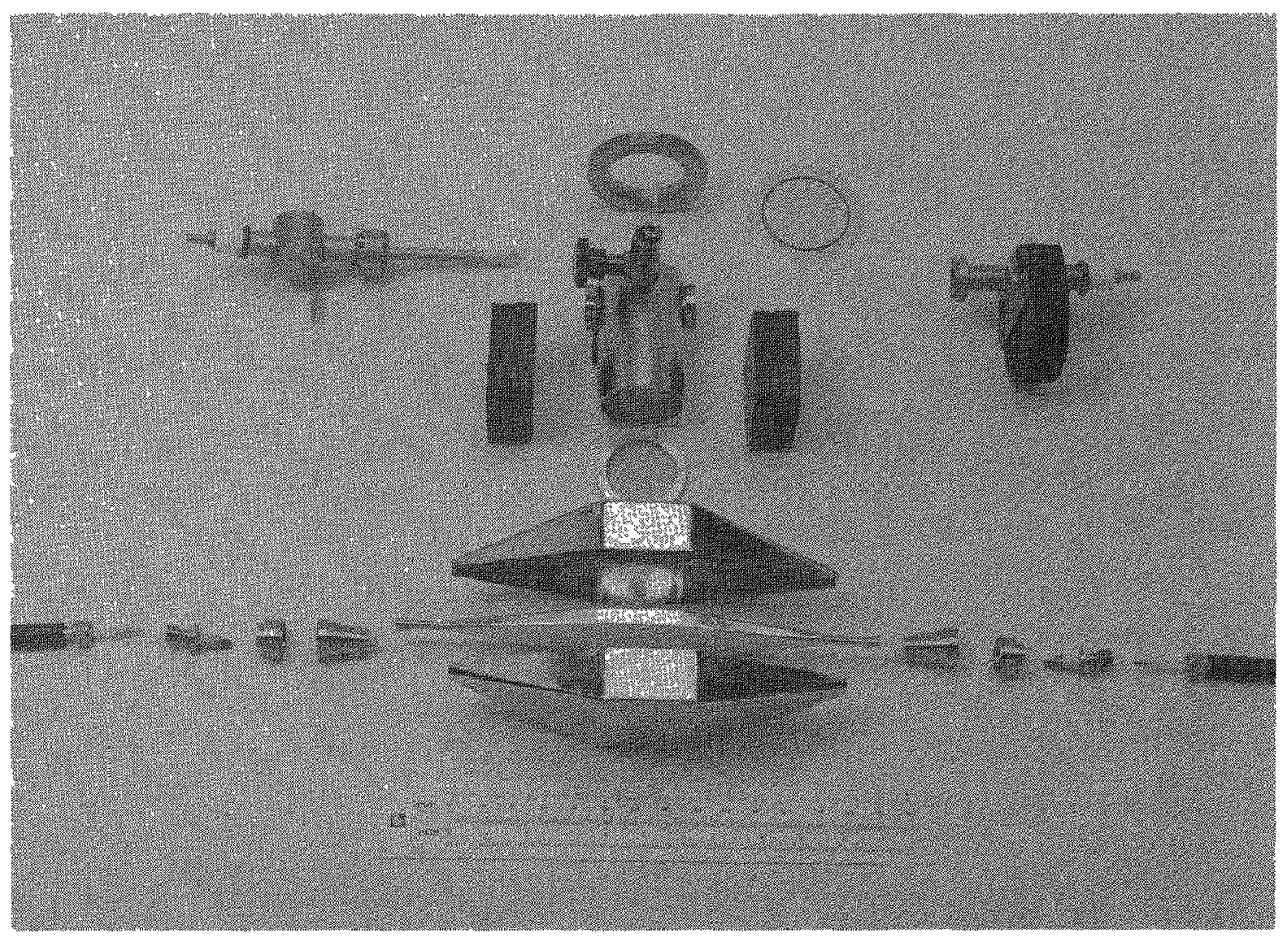

Figure 14. Components of a high-speed Compton diode ready for assembly. The unusual shape is necessary to maintain $50 \Omega$ geometry throughout the structure. The cathode and anode approximate confocal ellipses.

where $\phi_{\gamma}$ is the gamma-ray energy flux. It is desirable to measure $\alpha_{\gamma}$ over a long time interval. The gamma-ray measurement may cover the flux over 15 to 20 decades of output. This can only be done with several detectors that operate linearly over selected portions of the full output range. The detectors have different sensitivity, different sourceto-detector distances, different thickness lead attenuators, and different collimation. As illustrated in Fig. 6, each detector has several recorders. As mentioned previously, the thermonuclear reaction rate can be obtained by measuring nonelastic gamma rays produced in a converter 10cated close to the fusion source.

\section{Gamma-Ray Spectrometry}

The gamma-ray output spectra can also provide useful information about the performance of an exploding device. Nondispersive measurement of the fusion reaction rate is also important. Relatively high-energy gamma rays are produced directly in the several fusion reactions, which have very weak gamma-ray branching ratios. Since fusion gamma rays have well-defined energies, it is possible to differentiate these from other gamma rays.

Figure 15 schematically illustrates a gammaray spectrometer. It consists of a very thin beryllium foil located behind a beam-defining collimator, a magnetic field that bends Compton scattered electrons approximately $60^{\circ}$, and a detector array. The shape and orientation of the magnet are designed to collect, analyze, and focus electrons on a detector array located a safe distance from the high-flux region. Either Cerenkov detectors or Si PIN diodes measure the high-intensity electron bursts. The use of Si detectors sacrifices time resolution in favor of smaller size, thereby enabling 


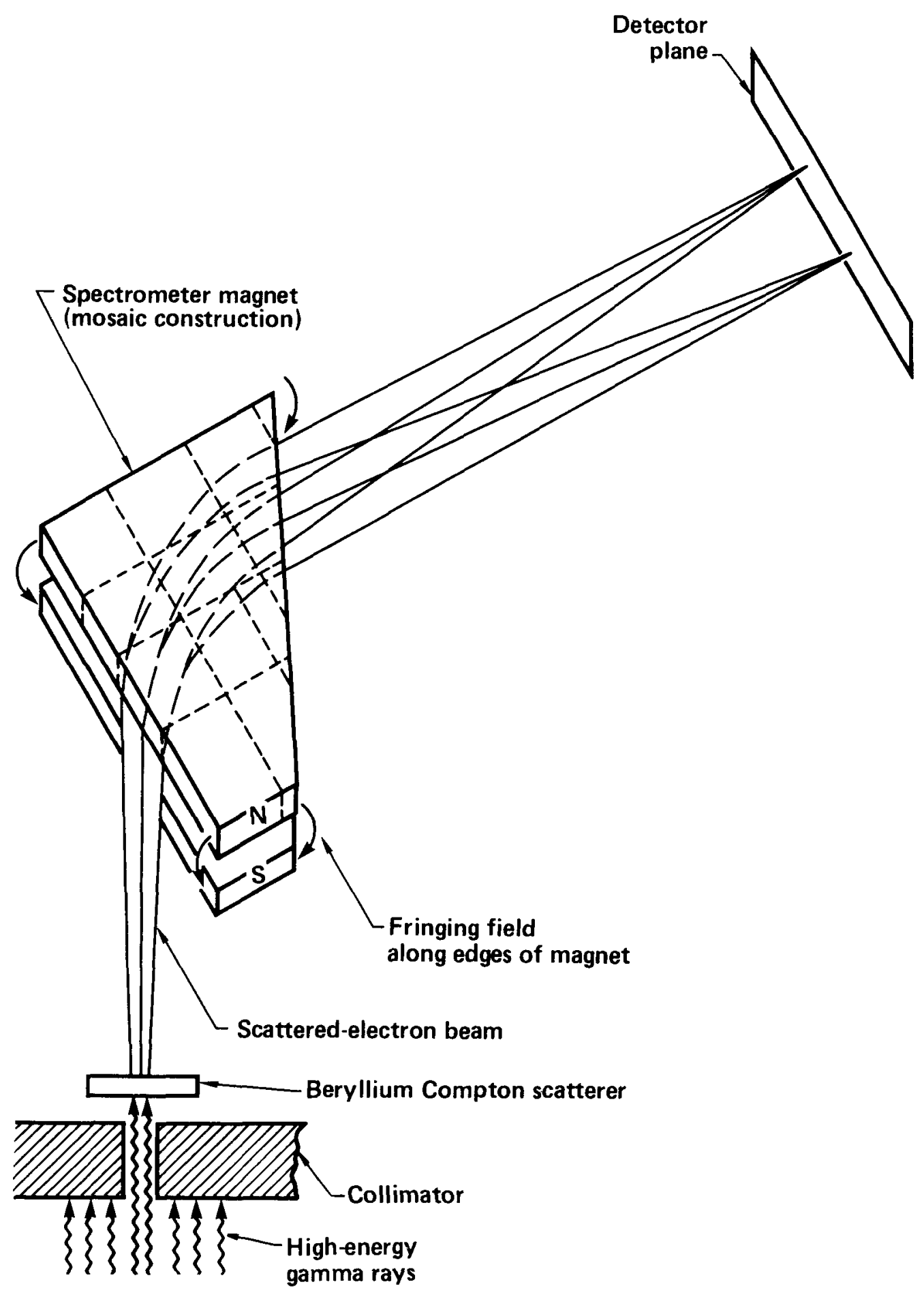

Figure 15. Schematic of a Compton electron spectrometer. Because there is a definite relationship between the photon energy and the angle of Compton electron emission, gamma-ray spectra can be measured with high-energy resolution. The energy covered is approximately proportional to the magnetic-field strength. Energy resolution depends on several parameters, including magnetic-field uniformity, foil thickness, and detector size. 
measurement at several energies. The gamma-ray energy measured is approximately proportional to the product of the magnetic-field strength and the radius of curvature.

Gamma-ray spectroscopy can also be accomplished via photonuclear reactions in ${ }^{9} \mathrm{Be}$ or ${ }^{2} \mathrm{H}$. In these reactions, which have thresholds at 1.7 and $2.2 \mathrm{MeV}$, respectively, photoneutron energy is determined using a time-of-flight neutron detector. The time dependence of the gamma-ray output is lost, however.

\section{X-Ray Diagnostics}

\section{Detection of X Rays}

In the context of nuclear-explosive diagnostics, $x$ rays lie in the $0.1-$ to $100-\mathrm{keV}$ energy range. $X$ rays arise from several different processes: bremsstrahlung, or acceleration of an electron in the field of the atomic nucleus, which gives a continuous spectrum; fluorescence $x$ rays, or radiative decay of excited atomic states, which give a discrete spectrum; and thermal $x$ rays, or photons naturally arising from emission of hot matter, which give a continuous spectrum. The $x$-ray emission from a nuclear explosive is predominantly thermal. A major reason for $\mathrm{x}$-ray measurements is determination of temperature. This generally requires some knowledge of the $x$-ray spectrum. Because the $x$-ray source is so bright $\left(\sim 10^{17} \mathrm{~W} / \mathrm{cm}^{2}\right)$ and because low energy photons interact so strongly with matter, $x$-ray detectors are seldom placed in the direct beam. Apertures, specular reflectors, scatterers, absorbers, or their combinations are needed to reduce the $x$-ray flux to a level where the detector will function properly. Except for clear apertures, these methods of flux reduction result in significant alteration of the $x$-ray spectrum, and appropriate corrections must be made. Soft $x$ rays $\left(E_{x}<10 \mathrm{keV}\right)$ are strongly attenuated by air. For this reason, $x$-ray measurements are accomplished in vacuum.

Several kinds of detectors can be used to measure transient flux in the $0.1-$ to $100-\mathrm{keV}$ energy range. They are much more sensitive than gamma-ray detectors and therefore can be substantially smaller. Among these are plastic scintillators, $\mathrm{Si}$ - and Ge-semiconductor diodes, and $x$-ray photodiodes. The $x$-ray photodiode is similar to the Compton diode in that photons generate a large electron current. The electrons have much lower energy than those produced by gamma rays and a bias voltage is required for efficient charge collection. Table 3 lists the important characteristics of these detectors. Except at very

Table 3. Characteristics of $x$-ray detectors.

\begin{tabular}{lcccc}
\hline \multicolumn{1}{c}{ Type } & $\begin{array}{c}\text { 1- to 100-keV } \\
\text { sensitivity } \\
\text { (electrons/ } \\
\text { keV) }\end{array}$ & $\begin{array}{c}\text { Response } \\
\text { time } \\
\text { (ns) }\end{array}$ & $\begin{array}{c}\text { Max. flux } \\
{[\mathrm{keV} /} \\
\left.\left(\mathrm{cm}^{2} \cdot \mathrm{s}\right)\right]\end{array}$ & $\begin{array}{c}\text { Max. } \\
\text { current } \\
\text { (A) }\end{array}$ \\
\hline $\begin{array}{l}\text { Plastic } \\
\text { scintillator }+ \\
\text { photomultiplier }\end{array}$ & $10^{4}-10^{6}$ & $5-8$ & $\sim 10^{20}$ & $\sim 0.1$ \\
$\begin{array}{l}\text { Plastic } \\
\text { scintillator }+\end{array}$ & $10^{2}-1$ & & & \\
$\begin{array}{l}\text { planar } \\
\text { photodiode }\end{array}$ & $1-100$ & -5 & $10^{17}-10^{19}$ & $\sim 10$ \\
$\begin{array}{l}\text { Silicon } \\
\text { X-ray } \\
\text { photodiode }\end{array}$ & $0.1-103$ & 1 & $10^{23}$ & 20 \\
\hline
\end{tabular}


low energy, they generally exhibit sensitivity which rapidly decreases with increasing energy. This behavior is similar to the energy dependence of photoelectric cross sections as shown in Fig. 12.

\section{X-Ray Spectrometry}

A hot body at temperature, $\mathrm{T}$, has spectral brilliance, $\mathrm{I}_{\mathrm{x}^{\prime}}\left(\mathrm{keV} / \mathrm{keV} \cdot \mathrm{cm}^{2} \cdot \mathrm{s} \cdot \mathrm{sr}\right)$ given by:

$I_{x}=\frac{C E_{x}^{3}}{e^{E_{x} / k T}-1}$

where $C$ is a constant of proportionality. This expression reduces to more simple forms: at low energy, $I_{x} \sim E_{x}^{2} k T$, and at high energy, $I_{x} \sim e^{-E_{x} / k T}$. Such spectral behavior can be exploited to obtain temperature as a function of time. Suitable energy intervals are selected and the intensity in each is measured absolutely. Selectivity is very important because the intensity has strong energy dependence.

One low-energy method employs a very thin scatterer of low atomic number, an appropriate filter, and an $x$-ray diode. The method, illustrated in Fig. 16, is very simple because a relatively large energy interval is selected. Photons are collimated with an aperture, scattered, filtered, and detected with an $\mathrm{x}$-ray diode. Below $1 \mathrm{keV}$, scattering efficiency increases with increasing energy, while detection efficiency decreases with increasing energy. The desired energy interval is accentuated by the choice of the filter material. For example, if copper were used, photons below the L-shell binding energy $(932 \mathrm{eV})$ would be preferentially transmitted to the detector. An $x$-ray diode with an Fe cathode has a sharp increase in sensitivity at its L-shell binding energy $(709 \mathrm{eV})$. This filterdetector choice defines the 709- to 932-eV energy interval of interest.

Low-energy spectrometers take advantage of the wave nature* of $x$ rays exhibited by reflection and diffraction. In the low-energy $x$-ray region, the refractive index of most substances is slightly below one. Because of this, there is total specular reflection for very small angles of incidence. The maximum energy which can be reflected, called

\footnotetext{
* $X$-ray wavelength in angstroms $\left(1 \mathrm{~A}=10^{8} \mathrm{~cm}\right)$ is given bv $\lambda=124 / E_{x}$, where $E_{x}$ is in $\mathrm{keV}$
}

the critical energy, is inversely proportional to the angle of incidence. For a given angle, $x$ rays above the critical energy are absorbed. Thus, a grazingincidence mirror serves as a low-pass filter. $X$ rays incident upon a crystal are diffracted when the Bragg conditions on wavelength, angle of incidence, and crystal plane spacing are satisfied. These are given by Bragg's law: $\mathrm{n} \lambda=2 \mathrm{~d} \sin \theta_{\mathrm{B}}$, where $\mathrm{n}$ is the order of diffraction, $\lambda$ is the $\mathrm{x}$-ray wavelength, $d$ is the distance between crystal planes, and $\theta_{B}$ is the angle of incidence and diffraction. A narrow range of wavelengths, determined by the crystal properties, is diffracted at each $\theta_{\mathrm{B}}$. Thus, a diffraction crystal serves as a band-pass filter. Figure 17 is a diagram of a crystal diffraction spectrometer. $X$ rays that pass through the collimating aperture are specularly reflected by the mirror and diffracted by a bent crystal toward an array of detectors. The mirror angle is made large enough to eliminate second-order diffraction of higher-energy $x$ rays. The crystal is bent to a concave cylinder. This gives a broader energy coverage and a convergence line where an anti-scattering slit and a filter are located. Behind the slit is an array of detectors. The angle and width of the detector defines the energy and the energy interval, respectively. Extraneous background is measured with a detector located out of the diffraction plane.

For $E_{x}>k T$, color temperature can be obtained by measuring the $x$-ray flux in a broad energy interval that is defined by a filter and a fluorescer, as illustrated in Fig. 18. The lower-energy bound is established by the large jump in $x$-ray absorption at the $\mathrm{K}$-shell binding energy of the fluorescer. The upper-energy bound is established by the large decrease in $\mathrm{x}$-ray transmittance above the K-shell binding energy of the filter. Emission of $\mathrm{K}$-fluorescence $\mathrm{x}$ rays is a signature of photoelectric absorption in the fluorescer. The fluorescence $x$ rays are detected with a filtered $x$-ray detector located out of the direct $x$-ray beam. To assure good energy discrimination, the fluorescence $x$-ray filter should be the same material as the fluorescer. For example, measurement in the $8.3-$ to $9.0-\mathrm{keV}$ energy range would require a copper filter, a nickel fluorescer, and a nickel fluorescence $x$-ray filter. Optimal energy response is achieved when the foils of these materials are approximately one mean-free-path thick for their characteristic $x$ rays. Several different filterflourescer measurements obtain time-dependent color temperatures. 


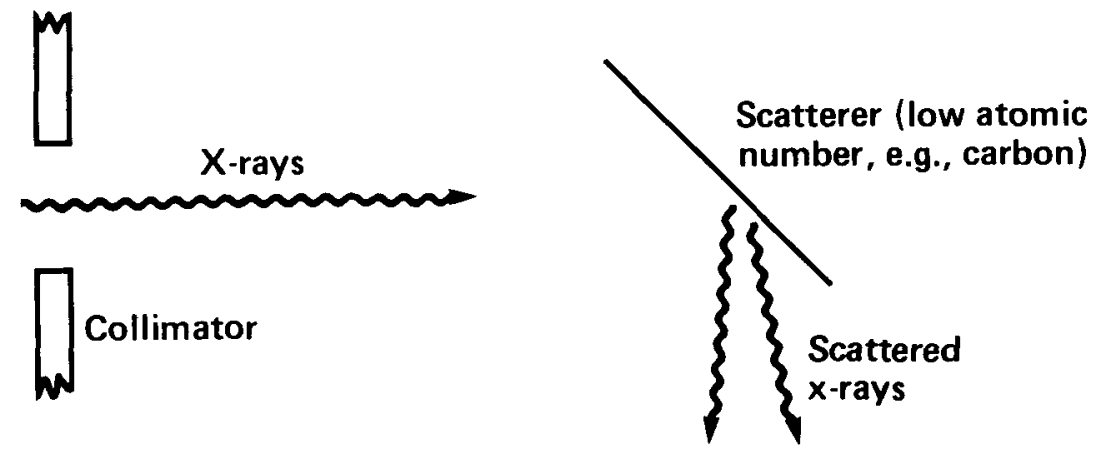

N

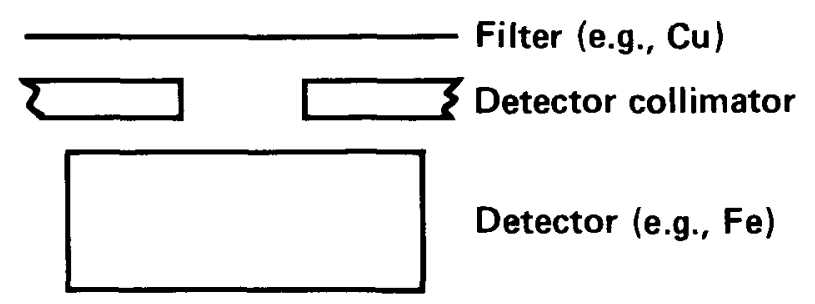

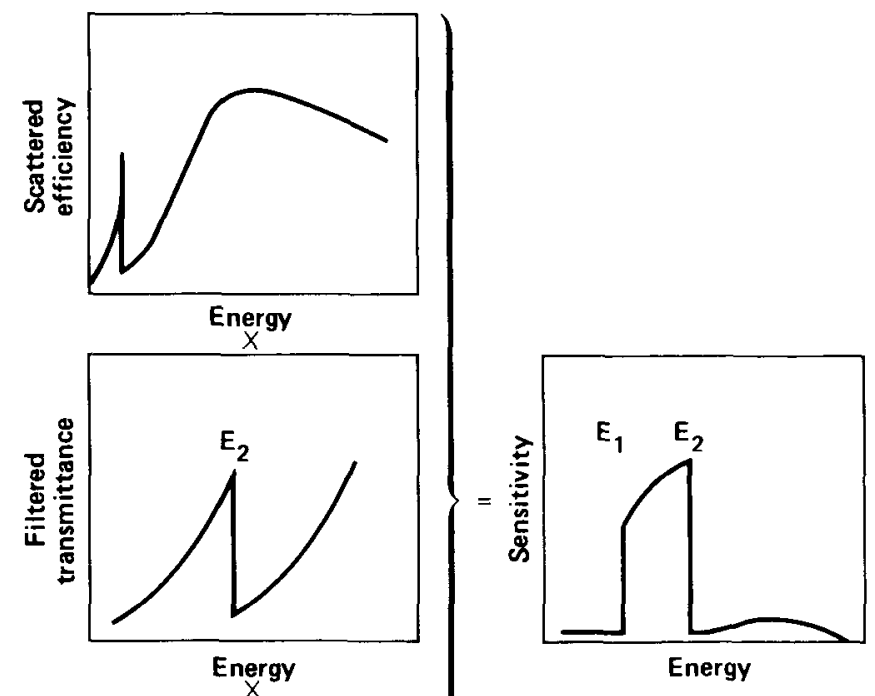

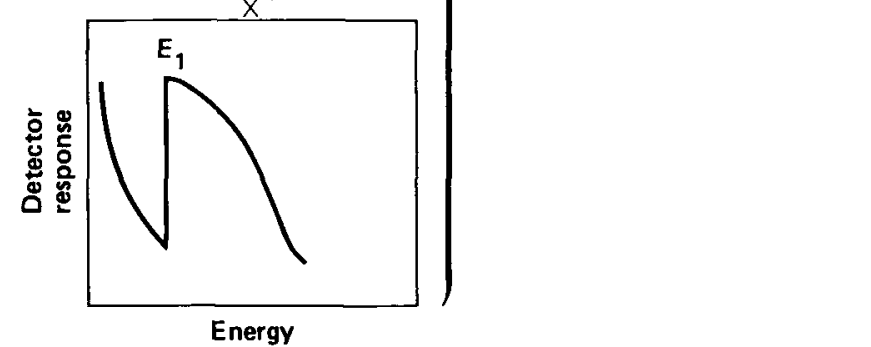

Figure 16. At low $x$-ray energy, color temperature is obtained with the scatterer-filter technique. Selectivity is achieved because of the energy dependences of scattering efficiency, filter transmittance, and detector response on photon energy. Energy resolution, given by the product of the responses of each element, is relatively poor. 

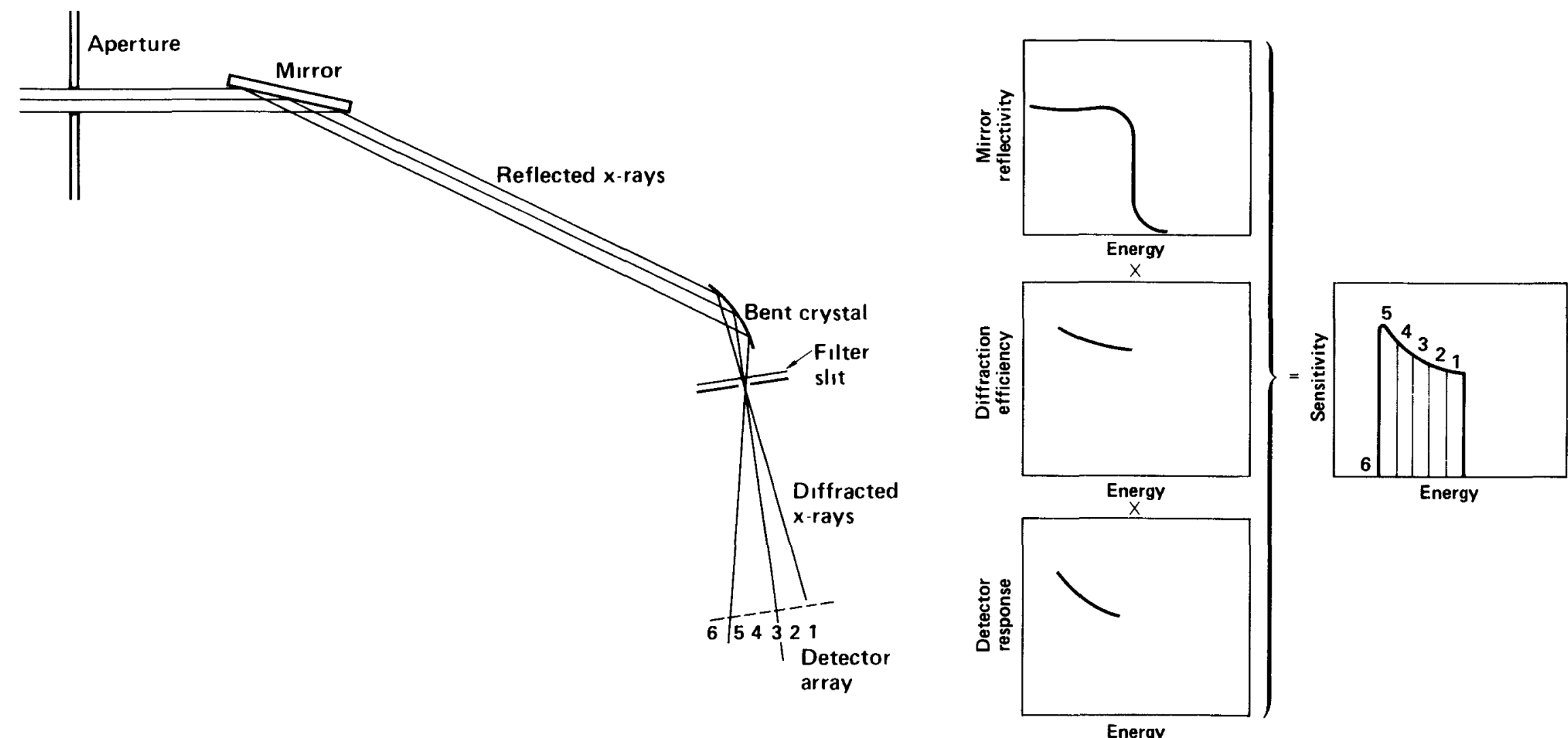

Figure 17. High-resolution $x$-ray spectrometry is accomplished with $x$-ray diffraction. In this low-energy spectrometer illustration, a specular reflector is used as a low-pass filter. In this way, contributions to the signals from higher-order diffraction are reduced considerably. The mirror is not necessary in a high-energy diffraction spectrometer. Energy resolution is given by the product of the responses of each element. 


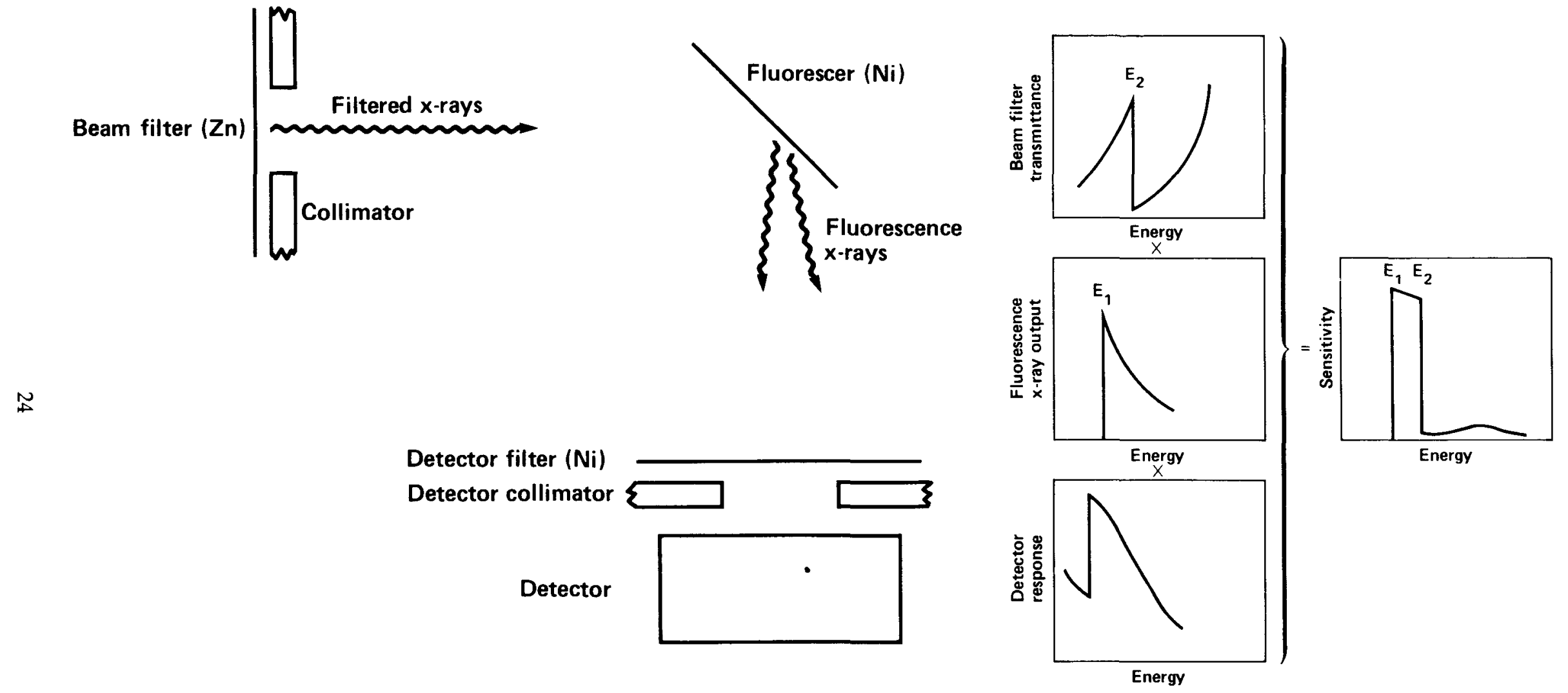

Figure 18. Color temperature is measured at high $x$-ray energy by a filter-fluorescer technique. Selectivity is achieved because of the sharp jump in absorption (and fluorescence $x$-ray emission) at the binding energy of the $n=1$ electron in each foil. Energy resolution, given by the product of the responses of each element, is relatively poor. 


\section{Imaging}

Multiparameter measurements are diagnostics in which output flux is simultaneously measured as a function of two independent variables, spatial coordinates, and time or energy. In contrast to the time-dependent, space average output measurements and low energy resolution spectroscopy discussed in the preceding sections, these diagnostics have much higher spatial or energy resolution and acquire substantially higher amounts of data. One of the performance questions, "Where were the radiations produced?" can be answered only with imaging diagnostics.

Because gamma rays and neutrons are so highly penetrating, images of the source must be formed using methods of geometric optics, as illustrated in Fig. 19. Neutrons from the source stream through the "pinhole" and produce an image in a plastic scintillator located a distance, $R$, from the source. The pinhole method imposes several practical problems. The mean-free-path of $14-\mathrm{MeV}$ neutrons in a heavy, dense element such as tungsten or gold is approximately $3.5 \mathrm{~cm}$. The "pinhole" should be several mean-free-paths thick to assure good image contrast. On the other hand, to obtain good spatial resolution, the "pinhole" diameter should be small. Thus, the actual pinhole is more like a small-bore tube than a pinhole. As a result, it must be located far from the source so that the field of view is sufficient. The image plane distance must also be large enough to assure that the scintillator light output is linear and that the blast does not destroy the cameras before the image can be recorded. This generally precludes obtaining good time resolution at the source, and therefore most neutron pinhole images are time-integrated. Because the pinhole has such a high aspect ratio, alignment is also very important. This problem can be avoided with an array of appropriately spaced pinholes which assures that one or more images will be formed. To account for uncertainty in predicted source strength, various pinhole diameters are used.

The radius of the smallest resolvable source element, $r_{s}$, is given by

$r_{\mathrm{s}}=\mathrm{r}_{\mathrm{PH}}(1+1 / \mathrm{M})$,

where $r_{P H}$ is the pinhole radius and $M$ is the magnification. $M=R_{2} / R_{1}$, where $R_{1}$ is the source-topinhole distance, $R_{2}$ the pinhole-to-scintillator

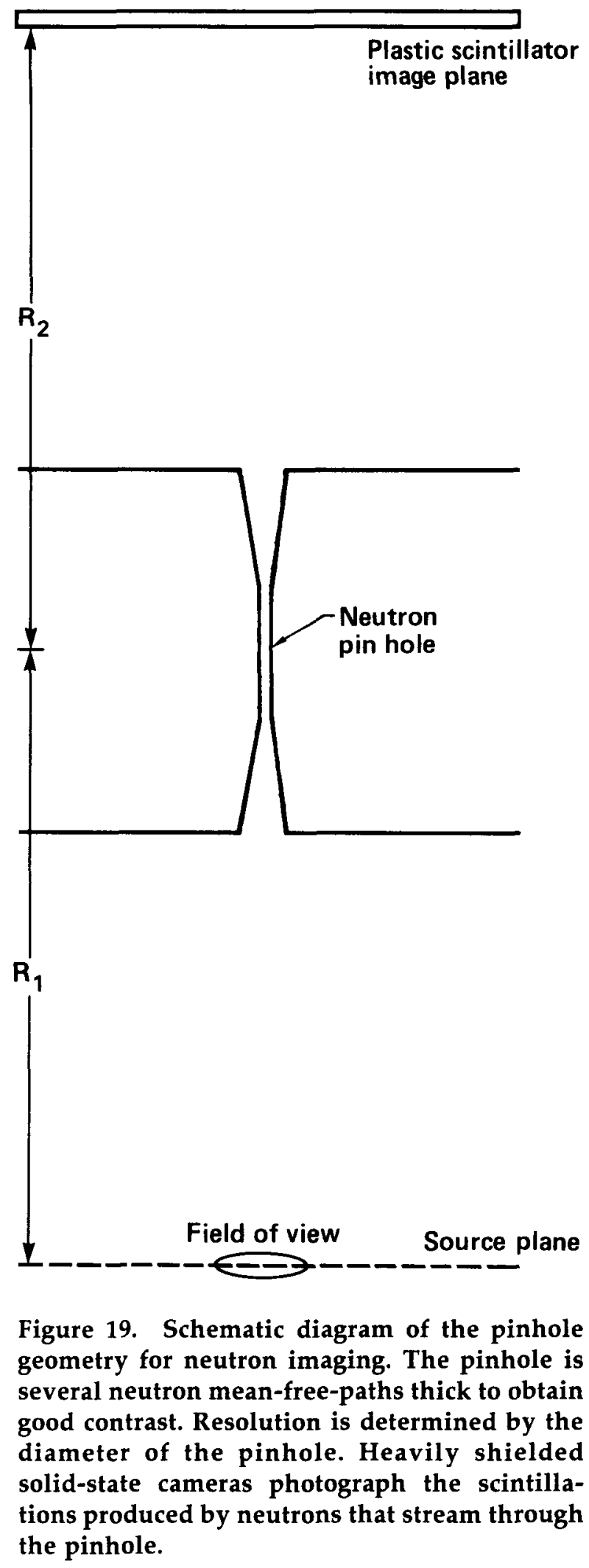

Plastic scintillator image plane 
distance, and $R=R_{1}+R_{2}$. The fluence at the scintillator, $\Phi$, is given by

$\Phi_{1}=\frac{\mathrm{Q}_{\mathrm{s}} \mathrm{A}_{\mathrm{PH}}}{4 \pi \mathrm{R}^{2}}$,

where $Q_{s}$ is the number of neutrons emitted per unit area and $\mathrm{A}_{\mathrm{PH}}$ the pinhole area. Neutron fluence must be less than $10^{11} \mathrm{n} / \mathrm{cm}^{2}$ to assure linearity of scintillation output. The imaging geometry and attenuation in the beam line are adjusted to keep the fluence below this value. Approximately $2 \times 10^{3}$ scintillation photons are produced per $\mathrm{MeV}$ absorbed by the plastic. The principal mode of energy deposition is ionization by elastically scattered protons in the plastic.
The plastic is $0.5 \mathrm{~cm}$ thick to restrict the loss of spatial resolution that is inherent in neutronproton scattering. To avoid internal reflections, it is painted black on one side. The scintillation light is focused onto an Si array which has at least 100 $\times 100$ resolution elements (pixels). The array can be turned on after the gamma rays have passed and is triggered to read out after the neutrons have safely passed. The charge accumulated in each pixel is amplified, digitized, and read out in approximately $2 \mathrm{~ms}$ ( $45 \mathrm{M}$ bits/s) on a single cable. The solid-state cameras have a dynamic range of approximately $10^{2}$. Several cameras focused upon one or more images obtain redundant coverage. The heavily shielded cameras are positioned in the backward direction to reduce extraneous background.

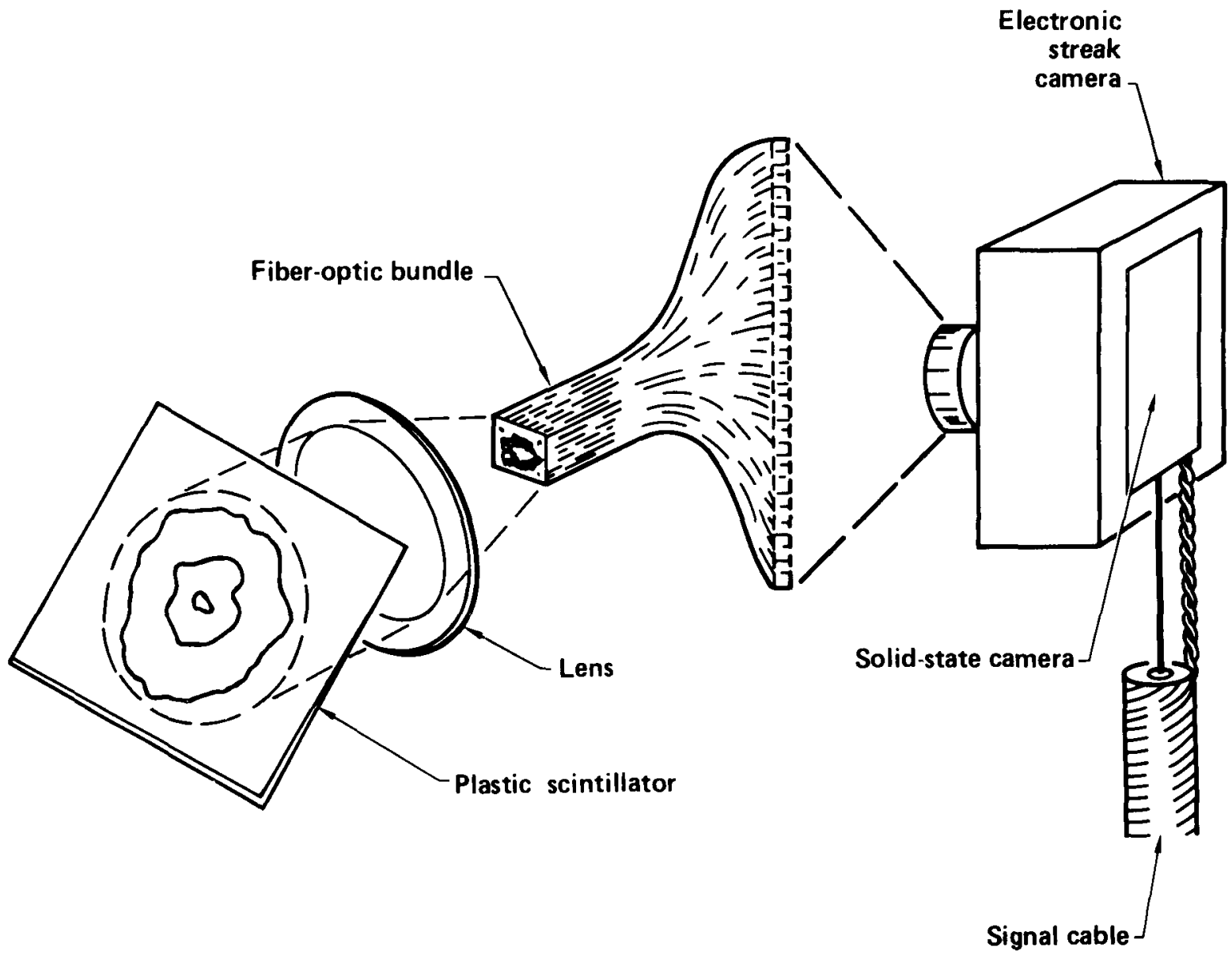

Figure 20. Streak-camera recording of spatially resolved neutron spectra. Scintillations are focused on one end of the image dissecting fiber-optic bundle and are piped to the streak camera, which records time-dependent scintillation intensity of each fiber. The streaked record is detected by a sensitive solid-state camera and read out digitally. Spatially dependent energy spectra are reconstructed with a computer. 
High time (or neutron-energy) resolution at the image can be obtained with image-intensifier tubes that operate as high-speed optical shutters. By sequentially shuttering several cameras, the neutron intensity is measured at several discrete times (energies) as a function of spatial coordinates. Such a measurement can also be accomplished with a fiber-optic image dissector as shown in Fig. 20. The scintillator image is focused onto the two-dimensional end of the array, and the one-dimensional end is coupled to a streak camera. Time (energy) is continuously recorded in the streak camera for each space point. Similarly, it is possible to obtain time-dependent $x$-ray spectra at high energy resolution with spatially coherent bundles of optical fibers.

\section{Conclusion}

A significant motive to provide more data of higher quality and lower cost is the expense of conducting underground experiments. The amount of data that can be transmitted to the recording systems has been steadily increasing as multiparameter diagnostics find wider application. Recent developments in electro-optics, high- speed electronics, and low-loss optical-fiber waveguides will accelerate this trend. In turn, this is leading to smaller detectors that are capable of achieving higher resolution in space, time, and energy, and to higher numbers of diagnostics experiments per test. 


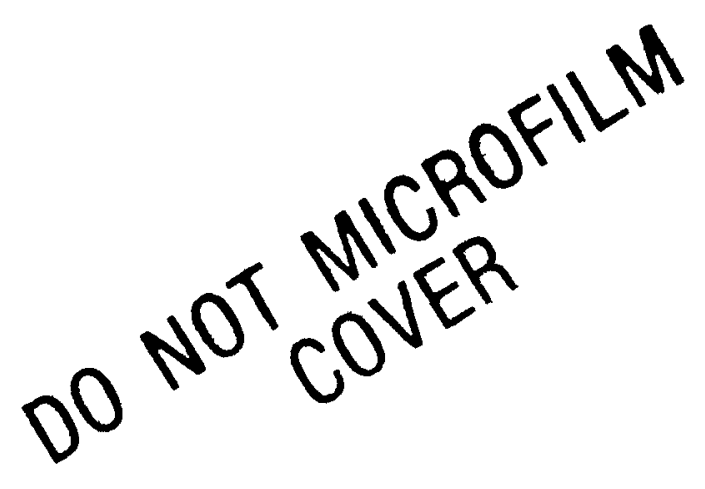

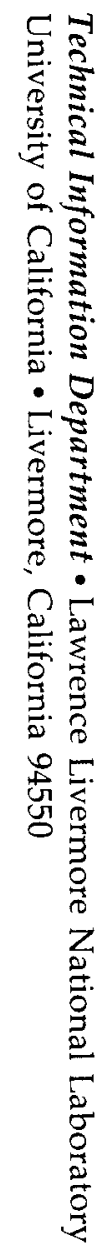

